\title{
Cladocères de quelques corps d'eaux du bassin moyen de l'Orénoque (Vénézuéla).
}

\author{
J. Rey! \\ E. Vasquez? \\ Mots clés : Cladocera, taxonomie, écologie, Vénézuéla, Orénoque.
}

L'examen de 110 échantillons pélagiques e1 de 16 échantillons littoraux de plusieurs corps d'caux du bassin moyen de l'Orénoque (lac de barrage, lacs d'inondation du système Orénoque et Caroni, rivières) a permis d'identifier 58 taxa de Cladocères, dont 24 sont nouveaux pour la faune vénézuélienne. 2 espèces, Alona ovata et Bosminopsis macaguensis, sont nouvelles pour la science. Alona karelica, élément paléarctique, n'avait jamais été mentionnée sur le continent américain. $35 \%$ de la faune étudiée sont représentés par des formes néotropicales, dont 8 endémiques sud-américaines.

Une partie taxonomique illustrée est consacrée à 13 especes rares ou peu connues.

La répartition des espèces dans les milieux prospectés et dans les deux catégories d'eaux blanches et d'eaux noires, a été étudiée. La faune la plus riche de Cladocères a été observée dans les lacs d'inondation de l'Orénoque, la diversité spécifique étant due aux formes littorales appartenant principalement à la famille des Chydoridae.

En milieu pélagique, les corps d'eaux blanches sont caractérisés par l'association Moina minuta, Ceriodaphnia comuta, Bosmina tubicen, Bosminopsis deitersi, les corps d'eaux noires par la dominance des Bosminidae : Bosmina hagmanni, Bosminopsis macaguensis, Bosminopsis deiterst, Ce riodaphnia cormuta. Le cas de Daphnia gessneri, géné ralement considérée endémique du système Amazone-Orénoque et présente dans un seul relevé, est discuté.

Cladocera of several water bodies in the middle basin of the Orinoco river (Venezuela).

Keywords : Cladocera, taxonomy, ecology. Venezucla, Orinoco.

The examination of 110 pelagic samples and 16 littoral samples from several water bodies in the middle basin of the Orinoco river (lakes formed by barrage or inundation of the Orinoco and Caroni river systems) revealed 58 taxa of $\mathrm{Clado}$ cera, of which 24 are new to the Venezuelan fauna. Two species, Alona ovala and Bosminopsis nacaguensis, are new to science. Alona karelica, a palatarctic species, has never been recorded before on the continent of America. $35 \%$ of the fauna studied are represented by neotropical forms, of which eight are endemic to South America.

An illustrated taxonomic section deals with 13 species that are rare or little known.

The distribution of species was studied in the environment examined and in the two categories of white and black waters. The richest cladoceran fauna was found in the lakes formed by inundation of the Orinoco, the species diversity being due to littoral forms, chiefly in the Family Chydoridae.

In the pelagic environment, white-water bodies were characterised by the species association Moina minuta, Ceriodaphnia comuta, Bosmina iubicen, and Bosminopsis deitersi, and black-water bodies by the dominance of Bosminidae : Bosmina hagmanni, Bosminopsis macaguensis, Bosminopsis deitersi, Ceriodaphnia comuta. The case of Daphnia gessneri, generally considered endemic to the Amazon-Orinoco system and present in a single survey, is discussed.

\section{1 - Introduction}

Les données taxonomiques, biogéographiques et écologiques sur les Cladocères néotropicaux sont très limitées (Collado \& al., 1984). Au Vénézuéla, la première étude réalisée est celle de Pearse (1921) sur le lac Valencia. En 1956, Brehm publie une importante liste d'espèces récoltées dans différentes régions de la Colombie et du Vénézuela et, à cette

1. Laboratoire d'Hydrobiologie, UA. CNRS 695 . Université Paul Sabat ier. 118, route de Narbonne. 31062 Toulouse Cèdex, France. 2. Fundaciòn La Salle de Ciencias Naturales, Apdo 51. San Felix. Edo Bolivar, Venezuela. occasion, pour la première fois est étudié du matériel provenant de l'Orénoque et de quelques lacs de son système d'inondation. A cette étude s'ajoutent celles de Brehm (1953, 1957), Margalef (1961), Herbst $(1967,1968,1975)$, et, plus récemment, les travaux d'Infante (1980) sur le lac Valencia et ceux de Zoppi de Roa (sous presse) sur un matériel des savanes inondables de Mantecal (Êtat Apure), situées dans le bassin hydrographique de l'Orénoque. L'immensité de ce bassin, la diversité des milieux aquatiques qu'il offre et le faible nombre d'études réalisées expliquent le peu de données actuellement disponibles sur ce groupe. Les Cladocères constituent cependant un élément important du plancton du 
système de l'Orénoque, comme l'ont montré les études réalisées sur un lac d'inondation de la section moyenne du fleuve et dans un de ses principaux tributaires : le Caroni (Vásquez \& Sánchez 1984, Vásquez 1984). Ils constituent, en outre, un élément prédominant dans le régime alimentaire de quelques espèces de poissons planctonophages de l'Orénoque et de l'Amazone (Carvalho 1984, Pérez 1984, Saint-Paul 1984).

L'objet du présent travail est de contribuer à la connaissance de la taxonomie des Cladocères peuplant la zone pélagique et littorale de divers corps d'eaux (rivières et lacs) du Bassin moyen de l'Orénoque. Dans le souci d'effectuer une première approche de la répartition de ces organismes, leur distribution dans les différents types d'eau (blanches et noires) aux différentes époques de l'année (conditions de basses et de hautes eaux) a été envisagée. Enfin, d'un point de vue appliqué, cette étude participe, à partir d'un inventaire, aux efforts actuellement effectués au Vénézuela pour la culture massive de Cladocères en vue de l'alimentation d'alevins et de poissons zooplanctonophages (Ravelo 1986).

\section{2. - Description générale de la région étudiée}

Le bassin de l'Orénoque est le plus étendu du Vénézuéla. Il totalise $950000 \mathrm{~km}^{2}$ dont $70 \%$ se situent en territoire vénézuélien. La longueur totale du fleuve est de $2140 \mathrm{~km}$ avec un débit moyen annuel de $30945 \mathrm{~m}^{3}$ $\sec ^{-1}$.

Dans la région du Haut-Orénoque, une partie des eaux du fleuve est apportée par le Bras Casiquiare au sub-bassin du Rio Negro, tributaire de l'Amazone.

Dans sa section moyenne, l'Orénoque coule à travers de basses plaines en donnant naissance à des lacs d'inondation dont la dynamique peut être expliquée brièvement : durant l'époque des hautes eaux (saison des pluies : Juin-Novembre) les plaines environnantes sont inondées. Plus tard, en période de sécheresse, les eaux se retirent au fur et à mesure de la baisse de niveau du fleuve pour se confiner dans les canaux du fleuve. Au cours de ce processus, les dépressions encore inondées restent isolées. Certaines constituent des corps d'eaux permanents, tandis que d'autres s'assèchent progressivement (milieux temporaires). Les rives des lacs présentent parfois des zones à macrophytes aquatiques d'extension variable. Les différences de niveau des eaux causent des changements notables et cycliques dans l'ecologie, la forme et la dimension des lacs. Ce cycle hydrologique se pro duit une fois par an.

Dans son tronçon moyen, l'Orénoque reçoit l'apport des eaux blanches par le nord et, par le sud, celui des eaux noires qui proviennent des hauts plateaux du bouclier guyanais (Mop 1969). Dans ce demier groupe, le Caroni constitue le fleuve des eaux noires le plus important avec un débit moyen annuel de $4100 \mathrm{~m}^{3}$

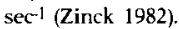

La classification, à partir des caractéristiques optiques, des différents types d'eaux trouvées dans le bassin de l'Orénoque (blanches, noires et claires) est fondée sur la classification proposée à la suite des travaux limnologiques effectués sur l'Amazone (Sioli 1950, 1951，1956, 1965). La relation qui existe entre la chimie de ces types d'eaux et les caractéristiques géologiques et géochimiques de leur région d'origine a été étudiée par divers auteurs. Le travail de Furch (1984) fourrit de nombreuses données sur ce point. Basées sur des caractéristiques optiques, les eaux blanches se différencient des eaux noires par leur fort contenu en sédiments en suspension. La couleur de ces dernières est due à sa haute teneur en composés humiques. Les eaux claires contiennent, comparativement, de basses concentrations en sédiments en suspension et en substances humiques. Néanmoins, il peut se présenter des formes intermédiaires entre ces types d'eaux (Schmidt 1972). Furch (1984) a réalisé une recherche exhaustive pour établir, d'un point de vue strictement chimique, une classification des différents types d'eaux de l'Amazone. Dans notre cas, les eaux noires du Caroni se différencient des eaux blanches de l'Orénoque par une concentration plus basse en matières en suspension, une conductivité plus faible, une teneur en nutrients également plus faible, une plus grande transparence et une coloration brunrougeâtre (Lewis \& Weibezahn 1981, Németh \& al. 1983. Vàsquez 1984, Vàsquez. \& Sànchez 1984).

\section{3. - Sites échantillonnés et méthodes}

\section{1. - Sites échantillonnés}

L'emplacement des stations est signalé dans les figures 1 et 2. L'échantillonnage a été effectué dans les corps d'eaux suivants :

- Huit lacs des eaux blanches du système d'inondation de l'Orénoque.

Une rivière des eaux noires. 


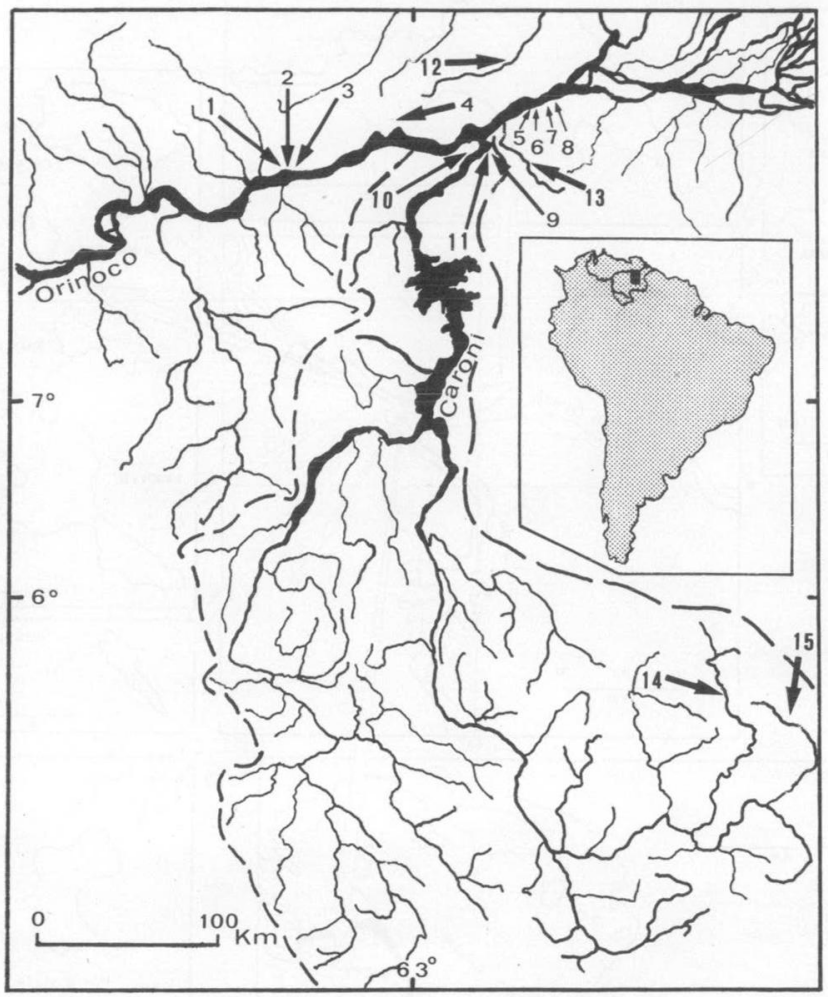

Fig. 1 : Localisation générale des stations d'échantillonnage. Lacs d'inondation du fleuve Orénoque : 1. Playa Blanca, 2. La Orsinera, 3. La Jobera, 4. Mamo, 5. Lagoven, 6. Rio Claro, 7. La Mariposa, 8. Los Castillos. Lacs d'inondation du fleuve Caroni : 9. Planta Modelo, 10. Punta vista. Lac de barrage sur le Caroni. 11. Macagua. Rivières : I2. Uracoa, 13. Upata : affluent de l'Orénoque. Affluents du Caronì : 14. Camoiran, 15. Yuruani. 


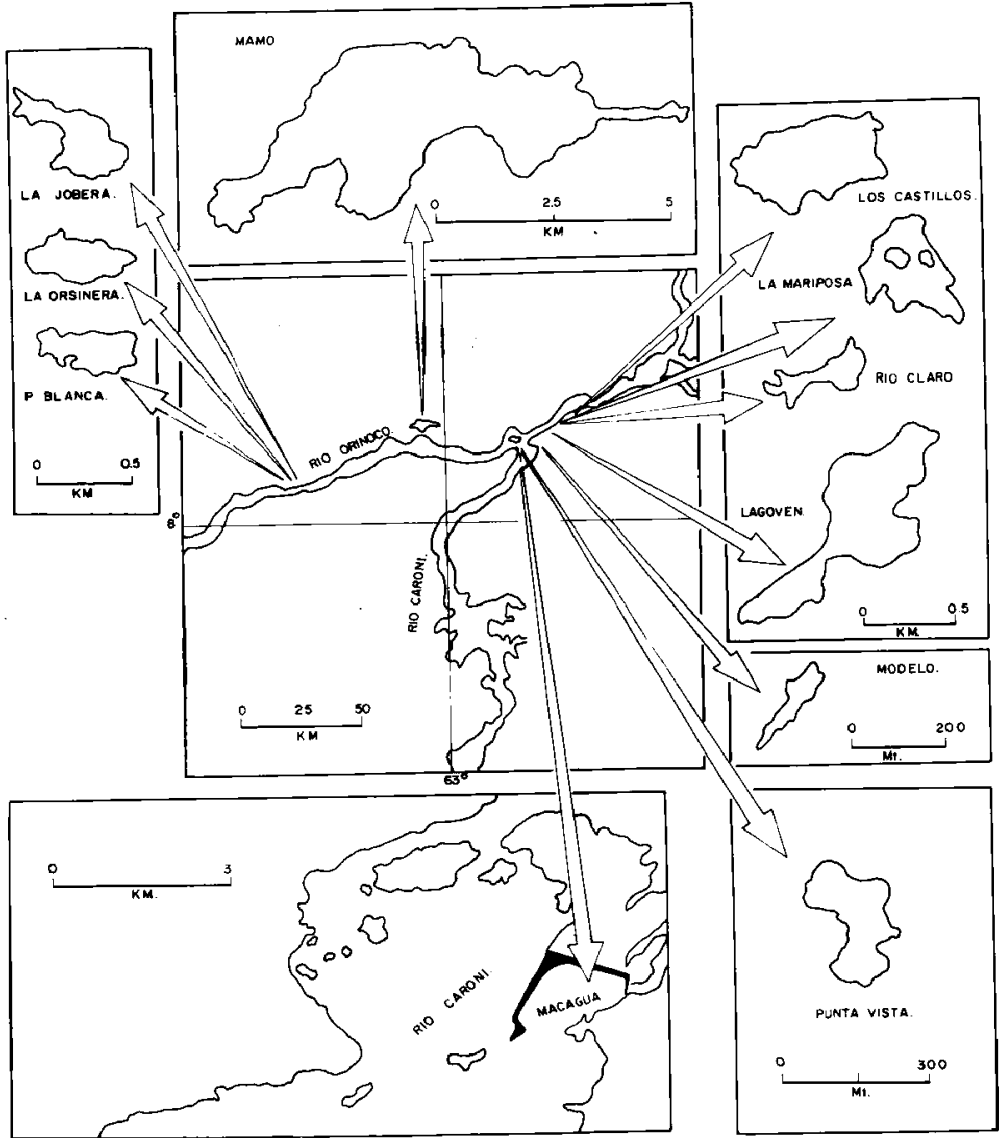

Fig. 2 : Localisation du lac de barrage sur le Caronì et des lacs d'inondation du système Orénoque. 
- Deux lacs d'inondation et un lac de barrage des eaux noires localisés dans la section basse du fleuve Caroni, affluent de l'Orénoque.

- Deux rivières des eaux blanches affluents du fleuve Caroni.

Un étang artificiel.

Pour chacune des stations sont donnés un code d'identification, les dates de récolte, le $\mathrm{pH}$, la température $\left({ }^{\circ} \mathrm{C}\right)$ at la conductivité $\left(\mu \mathrm{s}, \mathrm{cm}^{-1}\right)$ en valeur moyenne pour les dates de récolte. Pour les échantillons littoraux, le type de substrat végétal est indiqué.

\subsubsection{Lac des eaux blanches du système d'inondation} de l'Orénoque

LPB. Lac Playa Blanca (pH : 6,37; Temp. 29,6; Cond. 49,9). Dates des récoltes pélagiques: Année $1983: 13-3$, 19-10, 11-1t, 07-12. Année 1984:11-01, 16-02, 22-03, 25-04, 15-05, 14-06, 17-07, 23-08. Année $1985=17-01,10-04$.

Date des récoltes littorales : Année 1985 : 12-03 (Eichhornia crassipes).

LLO. Lac La Orsinera (pH : 6,6 ; Temp. 28,7 ; Cond. 33,7) ; Dates des récoltes pélagiques: Année $1983: 13-03,19-10$. 11-11. Anné 1984: 11.01, 16-02, 15-03, 25-04, 15-05, 14-06, 17-07, 23-08. Année 1985 : 28-08, 17-01, 12-02, 10-04. Dates des récoltes littorales: Année 1983: 27-04 (Paspalum repens).

Année 1985: 12.03 (E. crassipes).

LJO. Lac la Jobera (pH : 5,75; Temp. 29,3 ; Cond. 69,2) Dates des récoltes pélagiques: Année 1983 : 19-10, 11-11. Année 1984: 11-01, 16-02, 15-03, 25-04, 15-05, 14-06, 17-07, 23-08. Année 1985 : 17-01, 12-02, 13-03, 10-04.

Dates des récoltes littorales: Année 1985: 12-03 $(E$. crassipes).

LMA. Lac Mamo (pH : 6.39 ; Temp. 30,0 ; Cond. 13,3). Date des récoltes pélagiques: Année 1984: 05-07. Dates des récoltes littorales : Année 1983:10-10 (P. repens), 12-09 (P. repens et E. crassipes).

LLA. Lac Lagoven (pH : 6,31; Temp. 28,4 : Cond. 99,9). Dates des récoltes pélagiques: Année 1983: 18-10, 10-11, 06-12. Année $1984: 10-01,15-02,14-03,16-03,16-05,18-07$, 22-08. Année 1985: 16-01, 14-02, 13-03, 11-04.

Dates des récoltes littorales : Année 1983: 20-04 (E. crassipes). Année 1984: 07-09 (Nuphar sp., Salvinia sp.). Année 1985: 13-03 (Salvinia sp.).

LRC. Lac Rio Claro (pH : 7,39; Temp. 29,6; Cond. 477,7).
Dates des récoltes pélagiques: Année 1983 : 18-10, 10-11. 06-12. Année 1984:10-01, 15-02, 14-03, 24-04, 16-05, 15-06, 19-07, 22-08, 26-10. Année 1985: 16-01, 13-02, 13-03, 10-04.

LLM. Lac La Mariposa ( $\mathrm{pH}: 6,7$, Temp. 27,0; Cond. 200). Dates des récoltes pélagiques: Année 1983:09-08.

LLC. Lac Los Castillos (pH : 6,6; Temp. 27,2 ; Cond 40,3) Dates des récoltes pélagiques: Année 1983: 13-03, 18-10, 10-11, 06-12. Année 1984: 10-01, 15-02, 14-03, 24-04, 16-05, 15-06, 18-07, 12-08. Année 1985: 18-01, 13-02, 27-02, 13-03, 27-03, 10-04.

Dates des récoltes littorales ( $E$. crassipes dans tous les cas) : Année $1983: 14-04$. Année $1984: 24-12$. Année $1985: 28-02$, 13-03, 28-03, 30-05.

\subsubsection{Rivière des eaux blanches, affluent de l'Orénoque.}

RUP. Riviere Upata ( $\mathrm{pH}: 6,2 ;$ Cond. 200,0 )

Date de récolte pélagique: Année $1984: 31.07$.

\subsubsection{Rivière des eaux noires.}

RUR. Rivière Uracoa (pH: 5,7; Temp. 27,2; Cond. 33,4)

Date de récolte littorale : Année 1982 : 13.07 (E. crassipes).

3.1.4. Lacs dinondation et lac de barrage des eaux noires localisés dans la section basse du fleuve Caroni, affluent de t'Orénoque.

LPM. Lac Planta Modelo (pH : 6.8 ; Temp. 28,0; Cond. 144). Dates des récoltes pélagiques: Année 1983: 31-10, $10-11$. Date de récolte littorale : Année 1983: (Salvinia sp., Paspalum repens).

LMA. Lac Macagua (pH : 6,1; Temp. 27,4; Cond. 7,7) Dates des récoltes pélagiques : Année 1983: 10-05, 18-07, 10-08, 16-08, 30-09.

LPV. Lac Punta Vista (pH : 6,38 ; Temp. 29,0; Cond. 39,0) Dates des récoltes pélagiques: Année 1986:04-02, 04-03, 17-03, 01-04

\subsubsection{Rivières des eatu blanches, affluents du Caroni.}

RCA. Rivière Camoiràn (pH: 6,2).

Date de récolte pélagique: Année 1984: 18-08. RYU. Rivière Yuruani ( $\mathrm{pH}: 6,8$ ).

Date de récolte pélagique : Année $1984: 17.08$.

\subsubsection{Elang artificiel: EE.}

Dates des récoltes pélagiques: Année 1983:04-10. Année $1985: 12-03$.

\subsection{Matériel et méthodes}

Les collections pélagiques ont été effectuées au filet à plancton (vide de maille : $45 \mu \mathrm{m}$ ). 
Pour l'échantillonnage des organismes associés à la végétation aquatique émergente (Paspalum, par exemple) une masse de végétaux a été prélevée, lavée dans un récipient et finalement filtrée à travers une série de tamis (vide de maille minimal : $63 \mu \mathrm{m}$ ) afin de séparer les organismes des restes végétaux.

Pour la végétation aquatique flottante (Eichhomia. Salvinia, par exemple), la masse de végétation était prélevée à l'aide d'un filet conique ( $45 \mu \mathrm{m}$ de vide de maille) et traitée comme précédemment exposé. Tous les échantillons ont été fixés au formol à $10 \%$. Les Cladoceres ont été séparés et montés à la glycérine ou à l'alcool glycériné, en proportion variable selon la fragilité des organismes.

\section{4. - Liste systématique des espèces récoltées}

* Psetudosida ramosa (Daday, 1905)

Pseudosida bidentata Herrick, 1984

Sarsilatona serricauda (Sars, 1901)

* Latonopsis australis Sars, 1888

* Diaphanosoma birgei Korinek, 1981.

Diaphanosoma spinulosum Herbst, 1975

Diaphanosoma brevireme Sars, 1901.

Daphniidae

Daphnia gessneri Herbst, 1967

Simocephalus serrulatus (Koch, 1841)

Ceriodaphnia cornuta Sars, 1885

Scapholeberis sp.

Moinidae

Moina reticulata (Daday, 1905)

Moina mintta Hansen, 1899

Moina micrura Kurz, 1874

Moinodaphnia macleayi (King, 1853)

Bosminidae

Bosmina hagmanni Stingelin, 1904

Bosmina tubicen Brehm, 1953

Bosminopsis deitersi Richard, 1895

****Bosminopsis macaguensis n. sp. (1)

Macrothricidae

Macrothrix spinosa King, 1853

Echinisca triserialis (Brady, 1886)

Ilyocryptus spinifer Herrick, 1882

Grimaldina brazzai Richard, 1892.

Chydoridae

Chydorinae

Disparalona dadayi (Birge, 1910)

* Dunhevedia crassa King, 1853

Dunhevedia odontoplax Sars, 190.

Dadaya macrops Daday, 1898

Alonella excisa Fischer, 1854
*Alonella brasiliensis Bergamin, 1935

* *Alonella hamulata (Birge, 1878)

*Ephemeroporus tridentatus (Bergamin, 1939)

${ }^{\star}$ Ephemeroporus hybridus (Daday. 1905)

${ }^{*}$ Chydorus nitidulus (Sars, 1901)

Chydorus pubescens Sars, 1901

Chydorus eurvnoutus Sars, 1901

Aloninae

*Alona gunata Sars, 1862

*Alona guttata tuberculata Kurz, 1875

*Alona globulosa Daday, 1898

*Alona poppei Richard. 1897

*Alona quadrangularis /(O.F.M., 1785)

Alona monacantha Sars, 1901

*Alona eximia Kiser, 1948

*Alona rustica Scott, 1895

***Alona karelica Stenroos, 1897

****Alona ovala n. sp. (1)

Biapertura verrucosa (Sars, 1901)

Biapertura affinis (Leydig, 1860)

Biapertura karta (King, 1852)

*Biapertura intermedia (Sars, 1862)

Euryalona occidentalis Sars, 1901

"Camptocercus dadayi Stingelin, 1913 comb.

nov (2)

* Oxyurella ciliata Bergamin, 1939

${ }^{\star}$ Kurzia latissima (Kurz, 1875)

Kurzia longirostris (Daday, 1898)

Graptoleberis testudinaria (Fischer, 1848)

Leydigiopsis curvirostris Sars, 1901

Leydigiopsis omata Daday, 1905

*Acroperus of harpae (Baird, 1834)

****Espèce nouvelle pour la science.

***Espèce nouvelle pour le continent américain.

**Espèce nouvelle pour le continent sud. américain.

*Espèce nouvel le pour le Vénézuéla.

\section{5. - Étude taxonomique de quelques espèces}

Nous donnons ci-après, pour quelques espèces rares ou peu connues, une description illustrée des spécimens vénézuéliens, des remarques d'ordre taxonomique, et leur distribution sur le continent sudaméricain.

(1) Alona ovata n.sp. et Bosminopsis macaguensis n.sp. sont décrites séparément (Rey et Vàsquez, Annls Limnol., 22 (3) 1986).

(2) Rey et Vasquez, Annls Limnol., 22(2) 1986. 


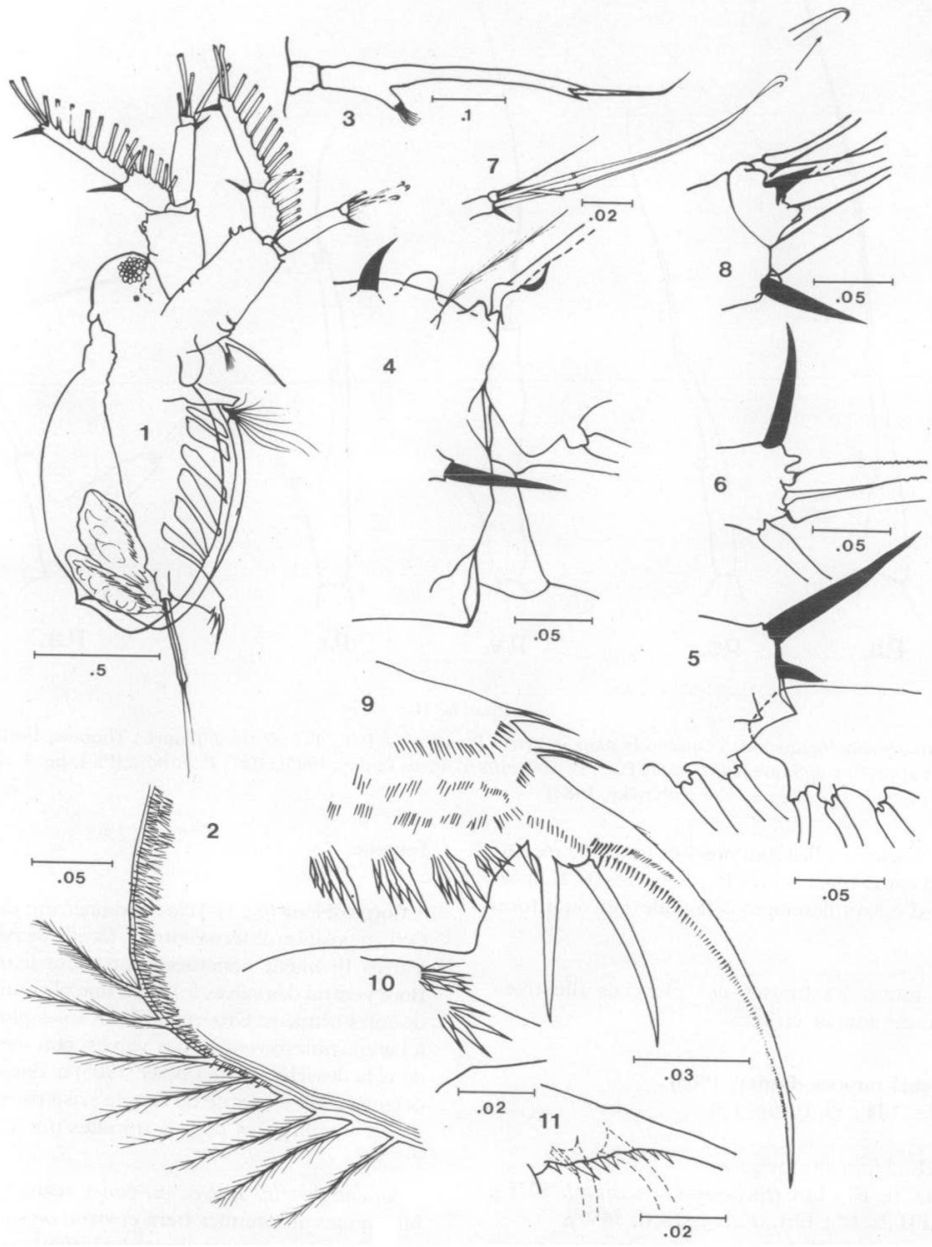

Planche I

Fig. 1 à 11 : Pseudosida ramosa (Daday 1905). Femelle parthénogénétique. 1 : vue générale latérale (segment distal des soies antennaires non figuré). 2 : angle postéroventral de la valve gauche, face interne. $3:$ antennule. $4:$ partie distale du basipode, face externe. $5:$ partic distale du ter segment de l'exopode. $6:$ partie distale de l'exopode. $7:$ soies de l'endopode (le segment distal de l'une des 3 soies terminales n'a pas été figuré). 8 : endopode : extrémité du Zème article et article terminal. 9 : partic distale du postabdomen. $10:$ groupes d'épines distales de la face ventrale du postabdo men. 11 : armature de la face interne de la griffe postabdominale. 


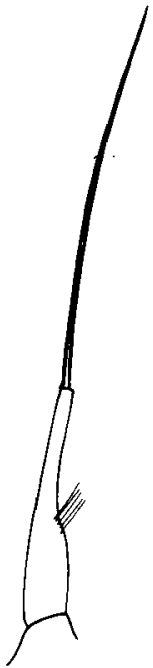

p.b.

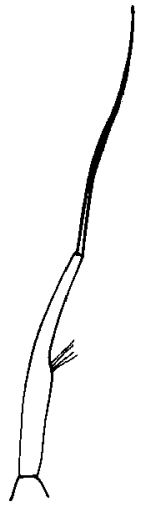

P.s.

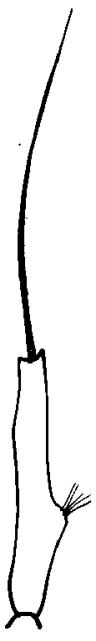

p.v.

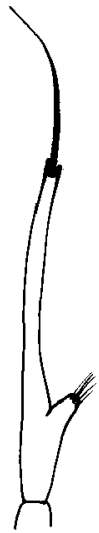

P.r.

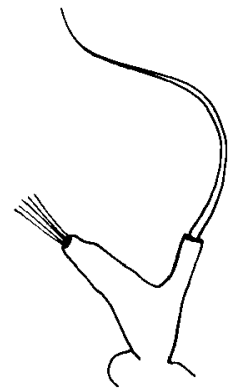

P.a.

\section{Planche II}

Variations morphologiques de l'antennule dans le genre Pseudosida. P.b.: P. bidentata (d'après Thomas, 1961); P.s. : P. szalayi (d'après Rey \& Saint-Jean, 1968) ; P.v. : P. variabilis (d'après Daday, 1905); P.r. : P. ramosa (Pl. I fig. 3, ce travail) ; P.a. : P. australiensis (d'après Korovchinsky, 1983).

Pour chacune d'elles sont précisés le site de récolte. le type d'eaux - blanches (E. Bl.) ou noires (E. No.) - et le mode d'échantillonnage - pélagique (Pél) ou lit toral (Litt).

Pour toutes les figures des planches illustrées, l'échelle est donnée en mm.

Pseudosida ramosa (Daday, 1905)

(Pl. I, fig. 1-14 ; Pl. II, fig. 1-5)

Matériel : 2 femelles ovigères

1 \& : LLC (E. Bl.), Litt. (Eichhomia crassipes) - $1075 \mu$

19 : LPB (E. Bl.), Litt. (E. crassipes) $1450 \mu$.

Décrite du Paraguay (Daday 1905) où elle fut retrouvée par Stingelin (1906), cette espèce rare et peu connue n'a, depuis, été mentionnée qu'une seule fois, du Brésil (Brandorff \& al. 1982).

\section{Femelle}

Corps oblong (fig. 1). Tête moyennement développée. Oeil en position antéro-ventrale. Ocelle de petite taille. Valves finement ponctuées sur toute leur surface. Bord ventral des valves formant une plicature et garni de soies sétulées. Groupe de 7 à 8 soies plus longues à l'angle antéro-ventral des valves, non mentionnées dans la description de Daday (1905) ni dans sa figure originale. Face interne de l'angle postero-ventral garnie de nombreuses petites spinules (fig. 2).

Antennule (fig. 3) avec un court segment basal se bifurquant au premier tiers environ de sa longueur, pour former une courte branche latérale sur laquelle sont insérées 7 papilles sensorielles. L'autre branche, longue et grêle, porte à l'apex une soie sensorielle relativement courte. 


\section{Antennes bien développees.}

Basipode large (fig. 4), presque aussi long que l'exopode avec, dans sa partie distale, une épine aiguë qui dépasse nettement le $1^{\circ}$ article de l'endopode. Région dorso-distale du basipode avec une épine courte, large, fortement incurvée et plus distalement, un tubercule arrondi. A l'angle dorso-distal se trouve un second tubercule, plus petit, doublement mamelonné, auquel semblent correspondre 2 saillies et 1 fossette situées à la base de l'exopode (systeme d'articulation ?). Dans la même région dorso-distale du basipode mais sur la face interne se trouve une longue soie, finement sétulée.

- Exopode développé. Segment proximal court et large, portant dans sa partie distale deux épines dont l'une est 4 fois plus courte que l'autre et un processus triangulaire (fig. 5). Epine terminale de l'exopode environ une fois et demi la largeur de ce segment (fig. 6).

- Endopode court avec les $1^{\circ}$ et $3^{\circ}$ articles réduits. Les deux soies les plus ventrales (articles 2 et 3). plus grosses et plus longues, terminées en crochet. La soie la plus dorsale, plus fine, est la plus courte (fig. 7). Epine terminale de l'endopode, petite, située entre les deux soies dorsales et la soie ventrale du $3^{\circ}$ article (fig. 8).

Formule antennaire : 6(2). 11(1)/0(0). 1(1). 3(1).

Postabdomen armé de 10 à 11 fascicules latéraux d'épines lancéolées (fig. 9). 2 groupes d'épines audessus de la griffe terminale ( $f$ ig. 10). Griffe terminale avec 3 épines basales, l'épine proximale étant environ 5 fois plus courte que les 2 suivantes. Face interne de la partie basale de la griffe (fig. 11) avec 7-8 denticules, courts et triangulaires.

Depuis le transfert de Pseudosida papuana Daday, 1900 dans le genre Sarsilatona récemment créé (Korovchinsky 1985), un caractère générique de Pseu. dosida est l'insertion latérale des papilles sensorielles sur le corps de l'antennule (Korovchinsky, p. 402, op. cit.).

Ce caractère fondamental étant respectè, l'antennule montre néanmoins à l'intérieur du genre une grande diversité de structure avec essentiellement 1 type non bifurqué ( $P$. bidentata, $P$. szalayi, $P$. variabilis) et 1 type bifurqué par développement plus ou moins grand d'une protubérance latérale portant les papilles sen. sorielles (pl. II. fig. 1-5). Pseudosida ramosa (Daday, 1905), forme sud-américaine et $P$. australis
Korovchinsky, 1983, forme australienne, sont les 2 seules espèces du genre à présenter une antennule bifurquée. D'un point de vue évolutif, il est intéressant d'observer que cette structure semble s'être réaliscée selon 2 voies opposées : allongement du corps de l'antennule avec développement moyen de la protubérance latérale chez la forme sudaméricaine, raccourcissement considérable du corps de l'antennule mais grand développement de la branche latérale chez la forme australienne. Parallèlement, la soie sensorielle montre pour chacune de ces 2 espèces un développement inverse de celui du corps antennulaire. Les valeurs comparatives fournies par le tableau I soulignent les variations morphologiques de l'antennule au sein du genre Pseudosida.

\section{Répartition}

Amérique du Sud.

Paraguay (Daday 1905, Stingelin 1906) ; Brésil (Brandorff \& al. 1982); Vénézuéla (ce travail).

\section{Latonopsis australis Sars, 1888}

(Pl. III, fig. 1-14)

Matériel: 15 femelles parthénogénétiques.

$I Q:$ LPB (E. Bl) ; Litt. (Eichhomia crassipes);

$10 \bigcirc ९$ : LLC (E. Bl); Litt. (E. crassipes);

2 \% : LPM (E. No); Litt. (Salvinia sp., Paspalum repens);

3 १ ९ : E.E. ; Pél.

Longueur : $585 \mu-1300 \mu$

99 ovigères: longueur minimale observée : $715 \mu$.

Décrite à partir de boues séchées d'Australie, Latonopsis australis a été réétudiée récemment d'après des spécimens topotypiques (Korovchinsky 1983).

Le corps oblong (fig. 1) des individus vénézuéliens, la tête massive non nettement séparée du corps, les grandes soies sétulées du bord ventral et postérieur de la carapace, les 3 longues soies glabres de l'angle postéro-ventral des valves (fig. 2), les groupes de 5 à 7 fines spinules submarginales (fig. 3 ), le contour et l'armature du postabdomen (fig. 4, 5) correspondent à la forme typique. Les quelques discordances observées comparativement à la forme australienne concernent les 2 paires d'antennes.

Antennule

La longue soie sensorielle garnie de 15 à 16 sétules offre un aspect serraté (fig. 6) et les 8 à 9 papilles sensorielles sont insérées sur une protubérance de l'antennule bien visible en vue latérale (fig. 7). Une 
Tableau I. Variations morphologiques de l'antennule dans le genre Pseudosida.

(1) : rapport longueur du corps antennulaire à longueur totale de l'antennule (corps antennulaire + soie sensorielle).

(2) : rapport longueur soie sensorielle à longueur totale de l'antennule.

\begin{tabular}{|c|c|c|c|c|c|}
\hline & $\begin{array}{c}\text { P. bidentata } \\
\text { Herrick } 1884\end{array}$ & $\begin{array}{c}\text { P. szalayi } \\
\text { (Daday 1898) }\end{array}$ & $\begin{array}{c}\text { P. variabilis } \\
\text { (Daday 1905) } \\
(=P \text { bidentata?) }\end{array}$ & $\begin{array}{c}\text { P. ramosa } \\
\text { (Daday } 1905 \text { ) }\end{array}$ & $\begin{array}{c}\text { P. australiensis } \\
\text { Korovchinsky } 1983\end{array}$ \\
\hline Sources & Thomas (196I) & $\begin{array}{c}\text { Rey \& Saint-Jean } \\
(1968)\end{array}$ & Daday (1905) & cet article & Korovchinsky (1983) \\
\hline (i) & 0,43 & 0,50 & 0,43 & 0,66 & 0,29 \\
\hline (2) & 0,56 & 0,50 & 0,56 & 0,33 & 0,70 \\
\hline
\end{tabular}

telle structure se retrouve chez le Latonopsis brevire. mis décrit du Paraguay (Daday 1905 : Tab. 14, fig. 3) et mis par la suite en synonymie avec $L$. australis (Harding \& Petkovsky 1963), mais on ne l'observe ni chez la forme décrite par Sars (1888: Pl. 1, fig. 3) ni chez les spécimens topotypiques revus par Korovchinsky (fig. 4B, op. cit.).

Les antennes montrent plusieurs points de divergence, principalement au niveau du basipode dont la région distale présente non 1 mais 2 tubercules doublement mamelonnés (fig. 8-9) aux angles dorsal et ventral, et 1 soie finement sétulée (fig. 10-11), non mentionnée dans la littérature, sur sa face interne.

L'épine distale du basipode ( $f$ ig. 9-11) apparaît plus robuste et plus longue que chez la forme typique avec une extrémité non pas aigtıë mais en biseau. Le processus distal du premier article de l'exopode est nettement triangulaire (fig. 12).

Formule antennaire : 4(1). $7(1) \cdot / 0(0)$. 1(1). $4(0)$.

Aucun spécimen, même de grande taille, n'a montré plus de 4 soies au ler segment de l'expode.

Un dernier caractère de la forme vénézuélienne concerne la sculpture de la carapace qui, chez la plupart des individus, présente de nombreuses formations de petite taille et d'aspect pyramidal, disposées plus ou moins régulièrement (fig. 14).

Compte tenu de l'ensemble des particularités observées, on peut penser que les spécimens du Vénézuéla représentent soit un taxon différent de l'espèce australienne, soit une forme identifiable au Latonopsis breviremis paraguayen de Daday qui constituerait alors une espèce valide. Ce problème taxonomique ne pouvant être résolu que par l'étude comparative de ces diverses populations, nous préférons pour l'instant rattacher nos individus à Latonopsis australis en soulignant leurs caractères propres.

Répartition

En Amérique du Sud : Paraguay (Daday 1905 : Latonopsis breviremis); Argentine (Martinez de Ferrato 1967 : L. breviremis) ; Brésil (Richard 1897, Brehm \& Thomsen 1936 : L. breviremis; Brandorff \& al. 1982) Vénézuéla (ce travail).

\section{Dunhevedia crassa King, 1853}

(Pl. IV, fig. 1-7).

Matériel : 2 femelles parthénogénétiques

1 Q : LRC (E. Bl.), Pél. - $385 \mu$.

$1 \odot$ :RUR (E. No.), Litt. (Eich homia crassipes) $-360_{\mu}$.

Outre Dunhevedia odonitoplax assez largement répandue en Amérique du sud, signalée à Cuba (Orghidan \& Negrea 1970), le matériel contenait 2 spécimens de $D$. crassa, espèce caractérisée par un labre à bord antérieur convexe, dépourvu de dents. Le labre (fig. 6, 7) présente la structure typique. Il est seulement légèrement plus court et un peu plus émoussé.

Le contour de la carapace (fig. 1), la dent incurvée à l'angle postéro-ventral des valves (fig. 3), la forme et l'armature du postabdomen (fig. 4) n'offrent aucun caractère particulier.

\section{Répartition}

Dunhevedia crassa, espece holarctique, connue également des régions éthiopienne, indomalaisienne et australienne, est à notre connaissance signalée pour la première fois en Amérique du Sud. Dunhevedia setigera (= Dumhevedia crassa ?) est citée du Chili (Richard 1897) mais il s'agit d'une simple mention sans figures. 


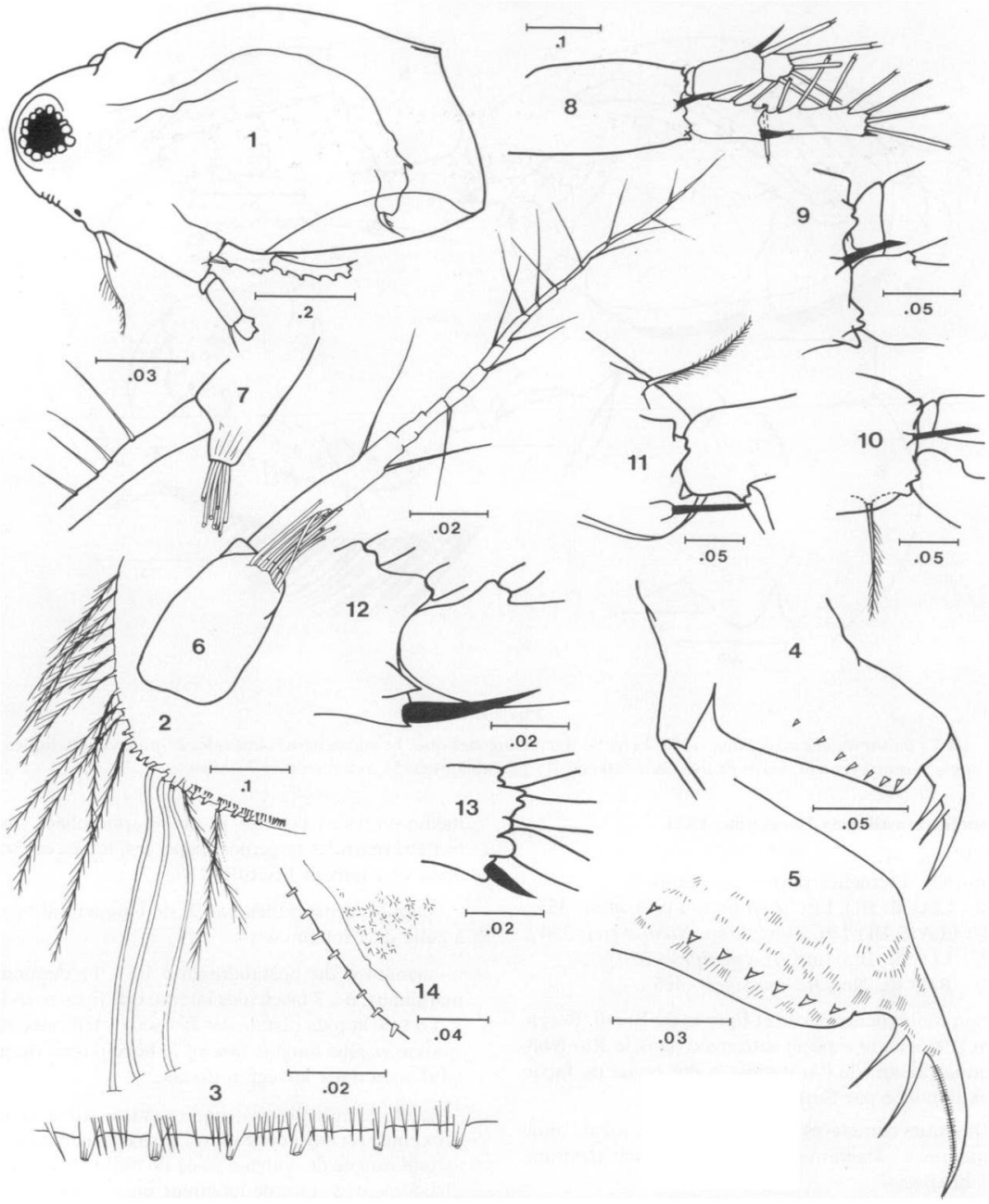

Planche III

Fig. 1 à $14:$ Latonopsis australis Sars, 1888. Femelle parthénogénétique . 1 : vue générale latérale. 2 : angle postéro-ventral, valve droite, face interne. 3 : spinulation du bord postérieur de la valve droite, face interne. 4 : postabdomen. 5 : partie distale du postabdomen. $6:$ antennule, vue postérieure. $7:$ antennule, vue latérale. $8:$ antenne (segment distal des soies antennaires non figuré). 9 et 10 : partie distale du basipode, vue externe. 11 : partie distale du basipode, vue postérieure 12 : ext rémité du ler article de l'exopode. 13 : partie terminale de l'exopode. 14 : sculpture des valves de la carapace. 


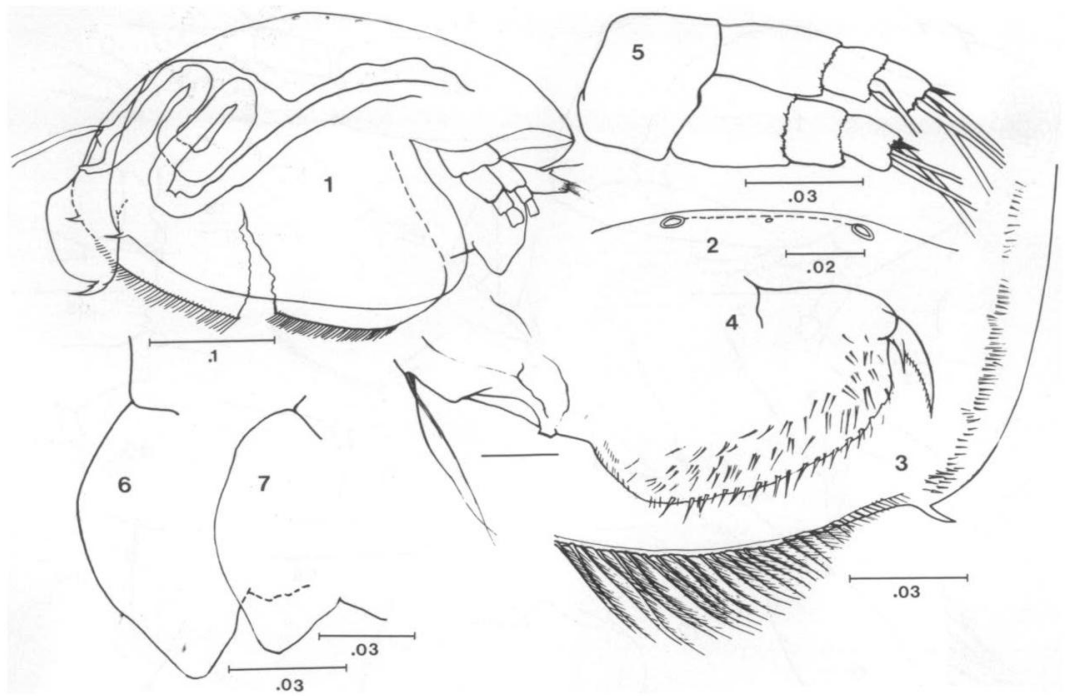

Planche IV

Fig. 1 à 7 : Durhevedia crassa King, 1853. Femelle parthénogénétique. 1: vue générale latérale. 2 : pores céphaliques. 3 : angle postéro-ventral, valve droite, face interne. $4:$ postabdomen. $5:$ antenne. 6 et $7:$ labre.

\section{Alonella brasiliensis Bergamin, 1935}

(Pl. V, fig. 1-12)

Matériel : 4 femelles parthénogénétiques.

1 \& : LLO (E. Bl.), Litt. (Eichhomia crassipes) - 355 ,

1 \& : LLA (E. Bl.), Litt. (Salvinia sp., Nuphar sp.) - $390_{\mu}$.

1 \& : LLC (E. Bl.), Litt. (E. crassipes) - $320 \mu$.

1 ९ : RUR (E. No.), (E. crassipes) - $405 \mu$.

Sommairement décrite et figurée du Brésil (Bergamin, 1935) cette espèce, retrouvée dans le Rio Nhamundà (Bassin de l'Amazone), a été revue de façon plus détaillée par Smirnov (1984).

Quelques caractères non notés pour la forme ama. zonienne, ou légèrement discordants, sont mentionnés ci-après :

- surface des valves avec une réticulation assez régulière de polygones finement ponctués (fig. 1).

- sétation du bord ventral des valves (fig. 3-6) répartie en 3 groupes : soies antéro-vent rales longues, soies médio-ventrales courtes et très rapprochées, soies postéro-ventrales largement espacées, toutes ces soies étant courtement bisétulées (fig. 7).

- papilles sensorielles (fig. 2) de longueur subégale à celle de l'antennule ;

- armature du postabdomen: 10 à 11 denticules marginaux, 6 à 7 fascicules latéraux de fines soies (fig. 8 ). La soje la plus distale des fascicules latéraux, plus épaisse et plus longue, atteint le bord dorsal du postabdomen dans la région distale.

- griffe terminale avec une ciliature sur la moitié proximale du bord concave et, sur sa face interne (fig. 9), une rangée de courtes soies de taille décroissante distalement. 3 soies de longueur inégale à la base de la griffe (fig. 10). Epine basale très longue, plus de la moitié de la longueur de la griffe terminale, légère. ment incurvée à son extrémité et, elle aussi, délicatement ciliée. 


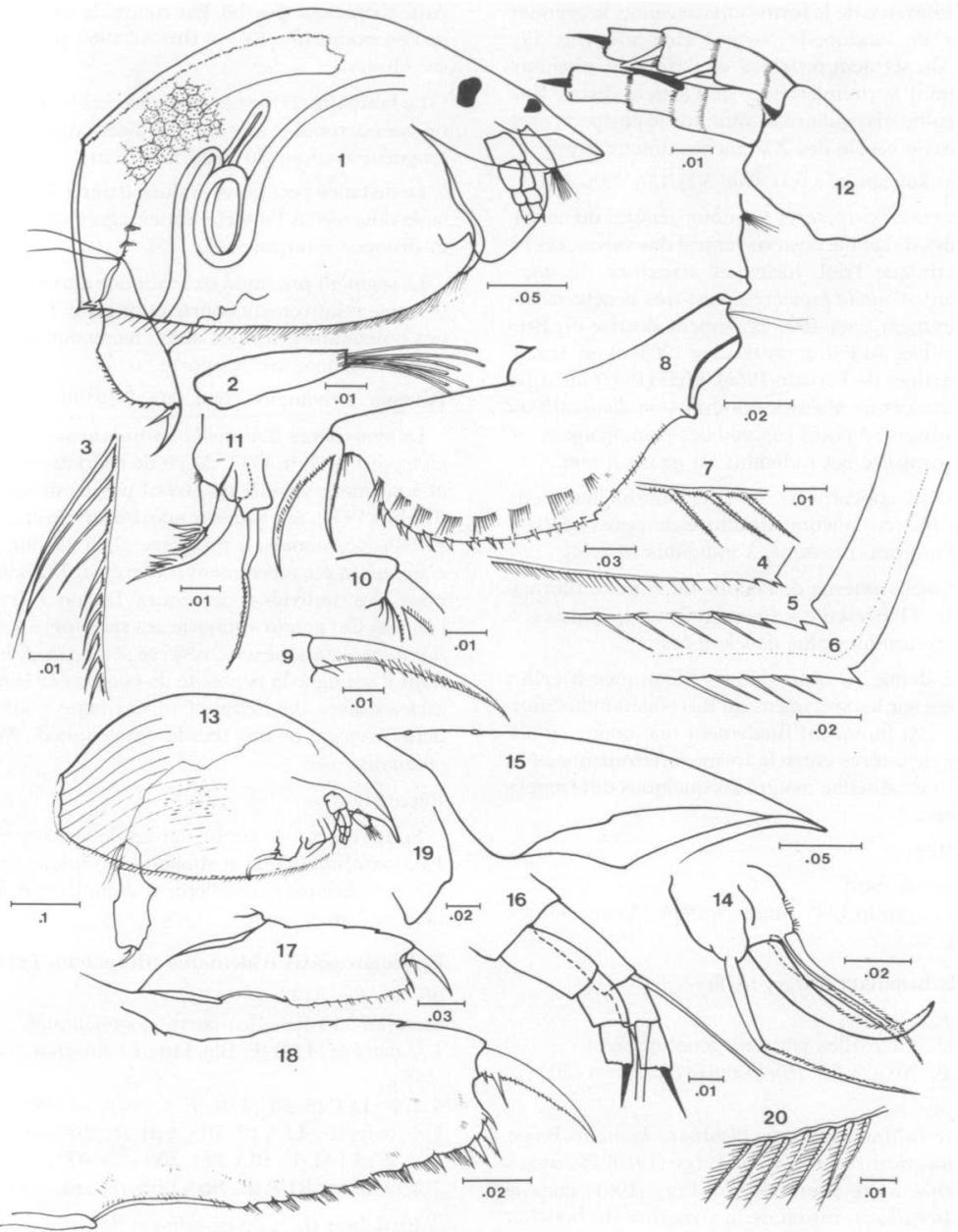

Planche V

Fig. 1 à 12 : Alonella brasiliensis Bergamin, 1935. Femelle parthénogénétique. 1 : vue générale latérale. 2 : antennule. 3 : bord antéro-ventral de la valve droite. 4 a $6:$ angle postéro-ventral des valves ( 5 et $6:$ valves droite et gauche respectivement du même spécimen). $7:$ sétulation du bord postéro-ventral de la valve droite. $8:$ postabdomen. $9:$ face interne de la griffe postabdominale. $10:$ spinules basales de la griffe postabdominale. $11:$ exopodite et branche externe de lendite de l'appendice thoracique I. 12: antenne (soies terminales non figurées).

Fig. 13 à 20 : Alonella hamulana (Birge 1879). Femelle parthénogénétique. 13 : vue générale latérale. 14 : exopodite et branche externe de l'endite de l'appendice thoracique I. 15 : vue latérale du bouclier céphalique. 16: antenne (soies terminales non figurées). 17 et 18 : postabdomen. $19:$ labre. $20:$ sétation postéro-ventrale de la valve gauche. 
A la différence de la forme amazonienne, le premier segment de l'endopode porte 1 fine soie (fig. 12). L'épine du segment proximal de l'exopode, plus longue, atteint le premier tiers de l'article distal. Une courte épine triangulaire se situe sur le basipode près de la partie basale des 2 branches antennaires.

Formule antennaire: $0(1) .0(0) .3(1) \cdot / 1(0) .1(0) .3(1)$

Par certains caractères (contour général du corps, denticules de l'angle postéro-ventral des valves, ocelle plus petit que l'œil, forme et armature du postabdomen), Alonella brasiliensis est très proche d'Alonella dentifera Sars 1901 également décrite du Brésil, retrouvée au Paraguay (Daday 1905) et en Argentine (Martinez de Ferrato 1966). Idris (1983) identifie des spécimens de Malaisie comme Alonella dentifera mais il observe 3 pores céphaliques principaux et, de ce fait, rapporte ses individus au genre Alona.

En ce qui concerne le matériel vénézuélien, nous n'avons observé aucune structure de pores céphaliques ( 1 individu disséqué, 3 individus in toto).

La branche externe de l'endite de la patte thoracique I (fig. 11) présente seulement 2 soies, épaisses, à sétules proximales plus développées.

Ces 2 dernières observations identiques à celles effectuées sur les spécimens du Rio Nhamundà (Smirnov, op. cit.), indiquent finalement une bonne similitude de caractères entre la forme amazonienne et la forme vénézuélienne malgré les quelques différences observées.

\section{Répartition}

Amérique du Sud.

Brésil (Bergamin 1935, Smirnov 1984) ; Vénézuéla (ce travail).

\section{Alonella hamulata (Birge, 1878)}

(Pl. V, fig. 13-20)

Matériel : 2 femelles parthénogénétiques.

RUR (E. No.). Litt. (Eichhonia crassipes)-500 $\mu$, $535 \mu$.

Décrit du Massachussets, Pleuroxus hamatus Birge, 1878, plus tard renommé par Birge (1910) Pleuroxus hamulatus, a été transféré par Frey (1961) dans le genre Alonella en raison de la structure du bouclier et des pores céphaliques.

Les 2 individus vénézuéliens présentent nettement les caractères de la forme nord-américaine : ornementation typique des valves ( $f$ ig. 13) et forte soie en crochet de la branche externe de l'endite de la première patte thoracique (fig. 14). Par contre, la soie secondaire de l'exopodite (fig. 101, in Brooks 1966, p. 647) n'a pas été observée.

Le labre (fig. 19) apparaît légèrement différent avec un apex arrondi et surtout une indentation nette à milongueur environ du bord antérieur.

La distance post-pore, évaluée d'après la ligne ecdysiale observée à l'interférentiel, égale environ $1 / 3$ de la distance interpores (fig. 15).

Le segment proximal de l'exopode antennaire porte une soie relativement courte et fine (fig. 16). Les 2 épines antennaires distales sont chacune aussi longues que le segment qui les porte.

Formule antennaire : $O(0)$. $O(0) .3(1) / 1(0)$. $1(0) .3(1)$.

La synonymie d'Alonella hamulata avec Pleuroxus chappuisi Brehm, 1933, décrit de l'Afrique occidentale et également signalé du Brésil par ce même auteur (Brehm 1937), est souvent admise (cf. Smirnov 1871 , p. 240). Néanmoins, le problème de la validité du nom chappuisi a été récemment soulevé par Dumont (1981) pour des individus du Fouta Djalon (Afrique de l'Ouest). Cet auteur rapporte ses spécimens à Alonella hamulata, quoique avec réserve (Alonella cf. hamata), mais il souligne la nécessité de confronter le matériel africain avec du matériel topotypique pour établir définitivement ou infirmer la synonymie de Pleuroxus chappuisi.

\section{Répartition}

Sous réserve de confirmation de synonymie entre Pleuroxus chappuisi et Alonella hamulana, cette dernière espèce est nouvellement signalée sur le continent sud-américain.

\section{Ephemeroporus tridentatus (Bergamin, 1939)}

(Pl. VI, fig. 1.14)

Matériel : 13 femelles parthénogénétiques.

1 o ovigère : LPB (E. BL.), Litt. (Eichhomia crassipes) $345 \mu$.

4 Q $9:$ LLC (E. Bl.), Litt. (E. crassipes) $300 \mu$ à $350 \mu$.

1 \& ovigère : LLA (E. Bl.), Litt. (E. crassipes) - $325 \mu$.

6 Q $\%$ : LLM (E. Bl.), Pél. $300 \mu$ à $400 \mu$.

$1 ९$ ovigère : RUR (E. No.), Litt . (E. crassipes) - $350 \mu$.

En dehors de la description et de l'illustration sommaires de Bergamin (1939), les seules données disponibles pour cette espece brésilienne sont celles de Frey (1982), qui en a effectué une redescription approfondie à partir de spécimens topotypiques. C'est à ce travail que nos individus ont été comparés. 


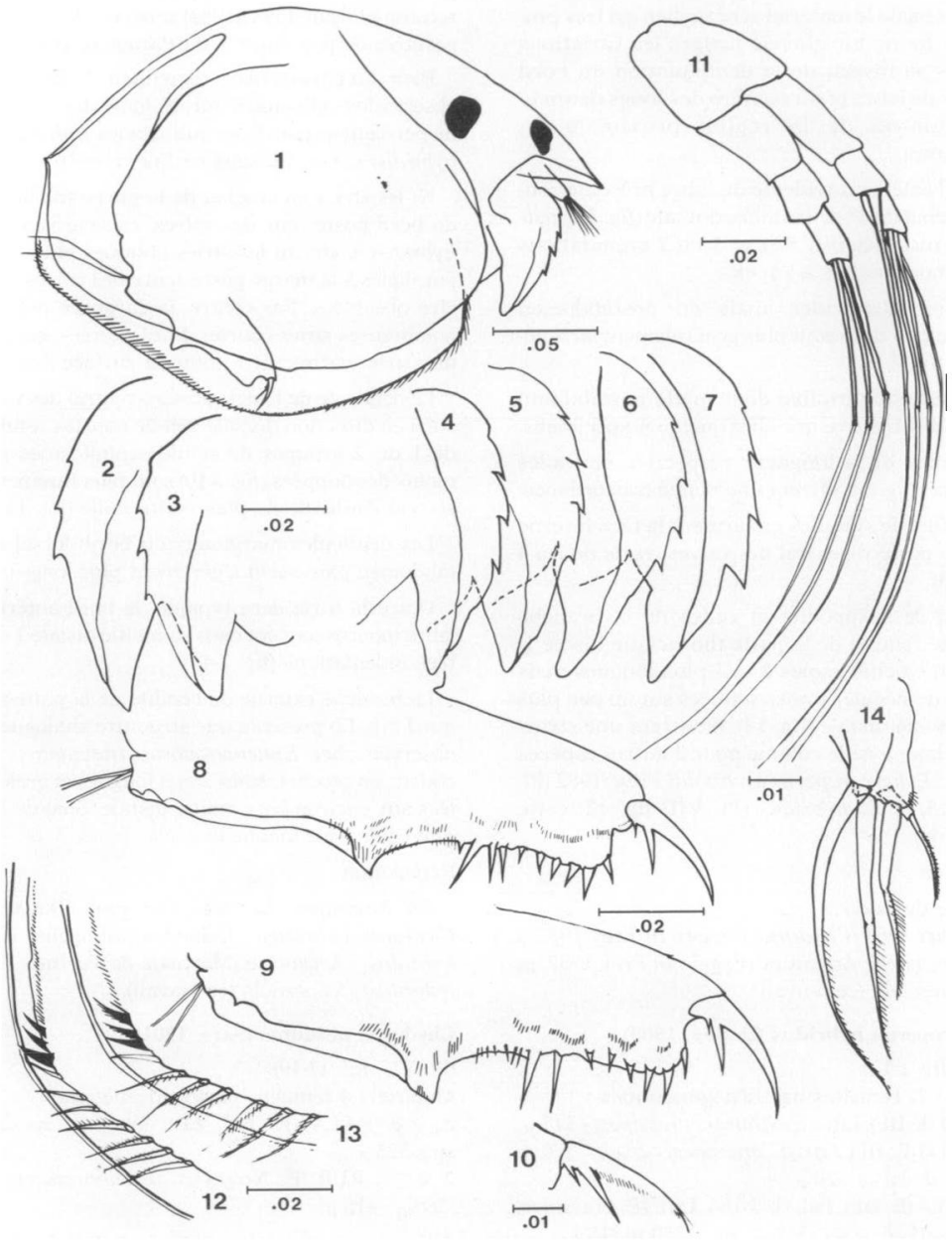

Planche VI

Fig. 1 à 14 : Ephemeroporus tridentatus (Bergamin, 1939). Femelle parthénogénétique. 1 : vue générale latérale. 2 à $7:$ labre. $8:$ antenne, $9:$ griffe postabdominale. 10 et $11:$ postabdomen. 12 et $13:$ angle postéro-ventral, valves gauches, face interne, de 2 spécimens. 14 : exopodite et branche externe de l'endite de la patte thoracique $\mathrm{I}$. 
Dans l'ensemble le matériel vénézuélien est très proche de la forme brésilienne malgré les variations observées au niveau de la denticulation du bord antérieur du labre et du nombre des longs denticules marginaux de la région proximale du postabdomen.

Le bord antérieur tridenté du labre présente souvent un début de $4^{\circ}$ dent antéro-dorsale (fig. 3-4) parfois bien marquée (fig. 5-6), et 1 ou 2 crénulations plus ou moins net tes à l'apex.

Les longs denticules anals du postabdomen varient de 2 à 3 mais sont plus généralement au nombre de 3 (fig. 8-9).

Le flagelle subterminal de la griffe postabdominale (fig. 10) n'a été vu que chez quelques spécimens.

Le nombre et la longueur respective des soies antennaires (fig. 11) offrent une bonne concordance.

Le nombre de spinules qui arment la face interne de l'angle postéro-ventral des valves, varie de 2 à 4 (fig. 12-13).

La soie de l'exopodite et celles de la branche externe de l'endite de la patte thoracique I (soie 1, courte, en crochet; soies 2 et 3 plus longues mais de longueur inégale), toutes sétulées sur un peu plus de leur moitié distale (fig. 14), montrent une structure similaire à celle connue pour 2 autres espèces du genre : Epheme roporus archboldi Frey, 1982 (Pl. IX, fig. 13) et E. hybridus (Pl. VII, fig. 12, cette publication).

Répartition

Amérique du Sud.

Brésil (Sars 1901 (Chydorus poppei, in Frey 1982); Bergamin, 1939); Argentine (Paggi : in Frey 1982, p. 246); Vénézuéla (ce travail).

\section{Ephemeroporus hybridus (Daday, 1905)}

(Pl. VII, fig. 1-12).

Matériel : 21 femelles parthénogénétiques.

1 o : LPB (E. Bl.), Litt. (Eich homia crassipes) - $315 \mu$.

4 १ : LLO (E. Bl.), Litt. (E. crassipes) $-2 \%$ 2 \%क ovigères $250 \mu$ à $320 \mu$.

99 १: LLA (E. Bl.), Pél. (1 ९ ov). Litt. (E. crassipes,

Salvinia sp.) -6 \% ? , 3 \% $\%$ ov. : 250 à $315 \mu$.

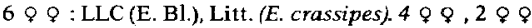
ov. : $285 \mu-320 \mu$

1 \% : LMA (E. No.), Pél. $230 \mu$.

Originellement décrite du Paraguay (Chydorus hybridus Daday, 1905), l'espèce a été redéfinie récemment par Frey (1982) à partir de l'étude comparative de populations du Paraguay et de Floride.

Bien qu'appartenant nettement à hybridus, les observations effectuées sur les individus vénézuéliens ne permettent pas de les rattacher à Ephemeroporus hybridus s. str., au sens de Frey (1982).

Ni les stries en crochet de la partie médio-dorsale du bord postérieur des valves, caractéristiques d' $E$. hybridus s. str., ni les stries obliques plus ou moins parallèles à la marge postérieure des valves, n'ont pu être observées. Par contre, la carapace présente de nombreuses stries courtes, fines, serrées, qui donnent un aspect vermiculé à toute la surface des valves.

Le denticule de l'angle postéro-ventral des valves est suivi en direction dorsale soit de courtes sétules, soit de 1 ou 2 groupes de sétules spiniformes plus ou moins développées (fig. 8-10) soit, plus rarement, d'un second denticule de plus petite taille (fig. 11).

Les denticules marginaux du bord dorsal du postabdomen paraissent légèrement plus longs (fig. 6-7).

Outre la forte dent typique, le bord antérieur du labre montre souvent dans sa moitié distale 1 ou 2 faibles indentations (fig. 24).

La branche externe de l'endite de la patte thoracique I (fig. 12) présente une structure analogue à celle observée chez Ephemeroporus tridentatus: soie 1 courte, en crochet, soies 2 et 3 longues et grêles, sétu. lées sur environ leur moitié distale. Soie de l'exopodite également longue et grêle.

Répartition

En Amérique du Sud: Paraguay (Daday 1905: Chydorus hybridus); Colombie (Stingelin 1904: C. hybridus): Argentine (Martinez de Ferrato 1967: C. hybridus); Vénézuéla (ce travail).

Chydorus nitidulus (Sars, 1901)

(Pl. VII, fig. 13-19).

Matériel : 4 femelles parthénogénétiques.

$29 \%$ : LLA (E. Bl.), Litt. (Nuphar sp, Salvinia sp.) $-325 \mu$

$29 \%$ : RUR (E. No.), Litt. (Eichhomia crassipes) $360 \mu-415 \mu$.

Comparés aux redescriptions de Paggi (1972) et de Frey (1982), les spécimens étudiés montrent peu de variations. En ce qui concerne la sétation du bord ventral des valves, les soies antéro-ventrales sont apparues beaucoup plus fines (fig. 13) que les 2 autres groupes de soies. 


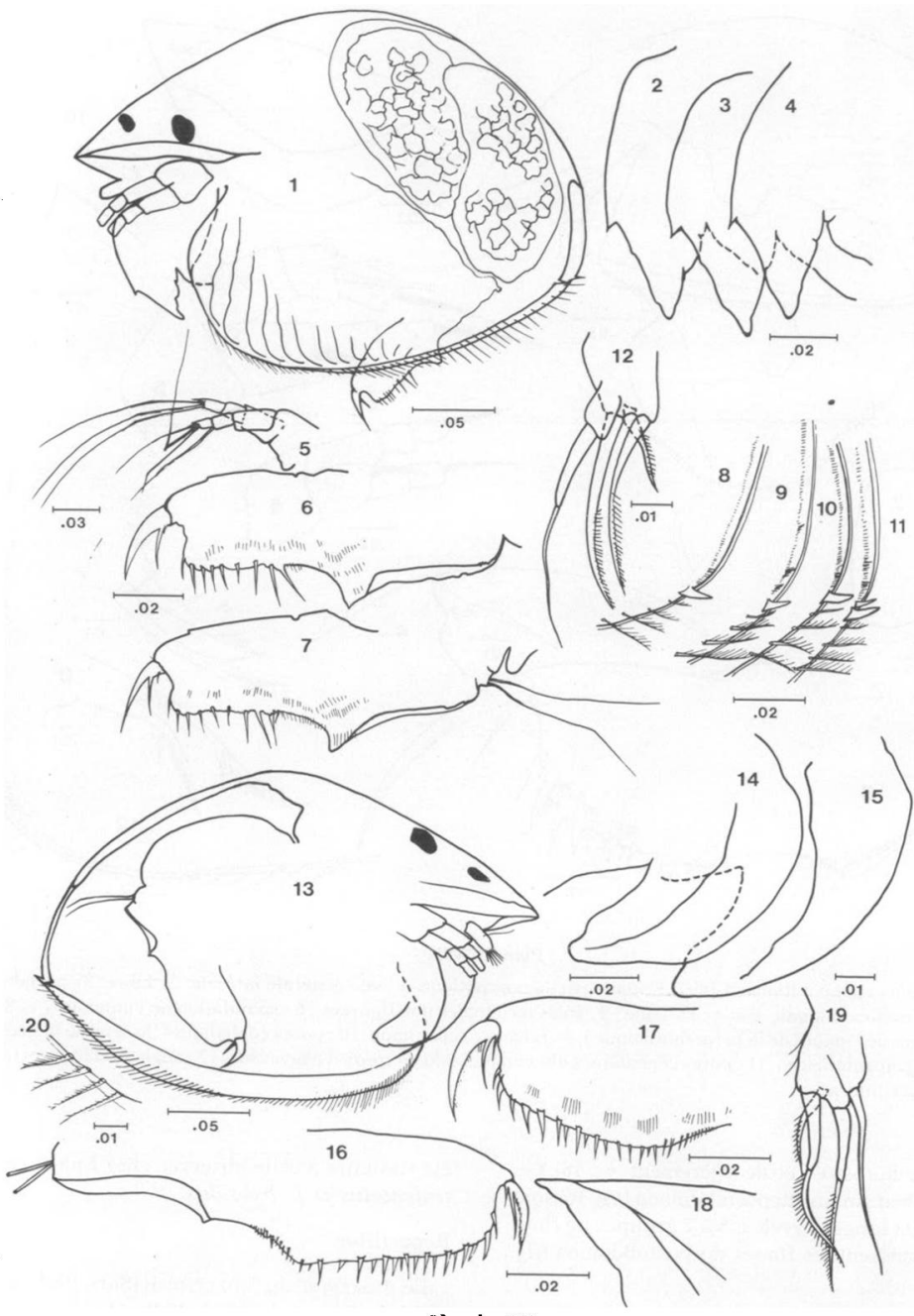

Planche VII

Fig. 1 à 12 : Ephemeroporus hybridus (Daday, 1905). Femelle parthénogénétique. 1 : vue générale latérale. 2 à $4:$ labre. $5:$ antenne. 6 et $7:$ postabdomen. 8 a 11 : angle postero-ventral, valves droites, face interne. 12 : exopodite et branche externe de l'endite de la patte thoracique $I$.

Fig. 13 à 19: Chydorus nitidulus (Sars 1901). Femelle parthénogénétique. 13 : vue générale latérale. 14 et $15:$ labre. 16 et 17 : postabdomen. 18 : région céphalique antérieure. 19 : exopodite et branche externe de l'endite de la pat te thoracique I. 20 : sétulation du bord postéro-ventral de la valve gauche, face interne. 


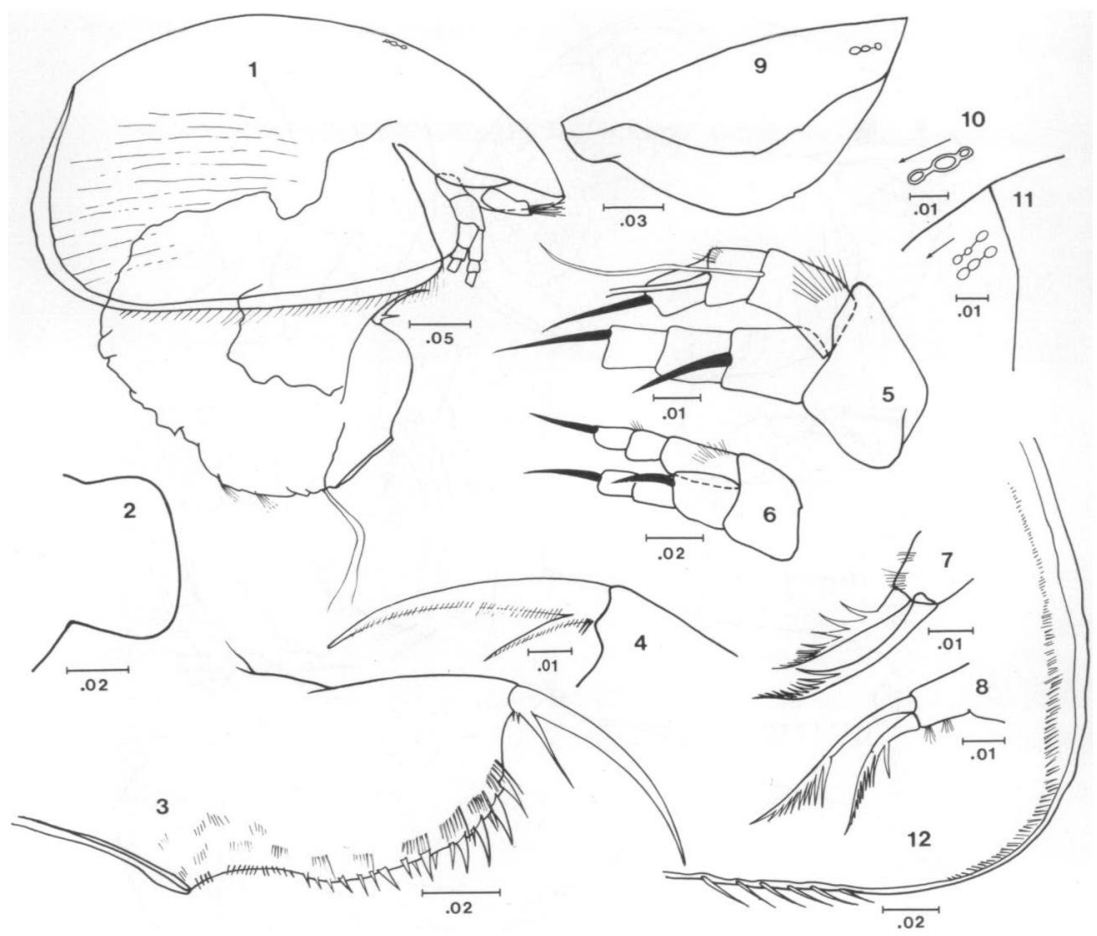

Planche VIII

Fig. 1 à 12. Alona poppei Richard 1897. Femelle parthénogénétique. $1:$ vue générale latérale. $2:$ labre. $3:$ postabdomen. $4:$ griffe postabdominale. 5 et 6 : antenne : 5 : soies terminales non figurées : $6:$ spinulation de l'antenne. 7 et $8:$ branche externe de l'endite de la patte thoracique I. $9:$ bouclier céphalique. 10 : pores céphaliques (le sens de la flèche indique la région antérieure). 11 : pores céphaliques chez un individu en cours d'exuviation. 12 : angle postéro-ventral, valve droite, face interne.

La pointe du rostre s'effile légèrement (fig. 18). Les denticules marginaux du postabdomen (fig. 16) sont relativement longs et grêles. 5 a 7 groupes de fines sétules garnissent les flancs du postabdomen (fig. 17).

La structure de la branche externe de l'endite de la première patte thoracique ( 1 soie courte et 2 soies de longueur inégale, sétulées sur leur moitié distale) est similaire à celle observée chez Ephemeroporus tridentants et $E$. hybridus.

\section{Répartition}

En Amérique du Sud : Brésil (Sars 1901 : Alonella nitidula, Bergamin 1939, $1940:$ A. nitidula); Paraguay (Daday 1905: A. nitidula); Argentine (Paggi 1972); Vénézuéla (ce travail). 


\section{Alona poppel Richard, 1897}

(PI. VIII, fig. 1-11)

Matériel : 3 femelles parthénogénétiques.

1 \& : LPB (E. Bl.), Pél. - $405 \mu$.

1 甲: LRC (E. Bl.), Pél - $405 \mu$.

1 Q : LLC (E. Bl.), Pél - $455 \mu$.

Décrite du Chili, citée des andes péruviennes (Delachaux 1918), du Brésil (Brehm 1937), de l'Ar-gentine (Olivier 1962) et, en Afrique, du lac Victoria (Delachaux 1917), cette espèce, peu connue, a été récemment retrouvée en Australie et figurée avec plus de détails par Smirnov (1983).

La forme générale du corps des individus du Vénézuéla, la forme et l'armature du postabdomen (fig. 1) sont en bonne concordance avec la description et les figures 37 et $\mathbf{3 8}$ de Richard (1897), à l'exception de la grande soie distale des fascicules latéraux qui, comme l'a observé également Smirnov (1983) pour la forme australienne, dépasse nettement le bord dorsal du postabdomen (fig. 3). Les autres caractères de l'armature postabdominale : sétulation de la dépression anale, ciliation de la griffe terminale (non figurés chez le spécimen aust ralien) sont conformes à la description originale. L'observation de la griffe terminale, à l'immersion, montre sur tout le bord concave une fine ciliature formant un peigne délicat de cils, plus longs dans le tiers proximal ( $f$ ig. 4). L'épine basale, grêle et aiguë, est également ciliée. 2 sétules s'insèrent à la base de la griffe postabdominale.

Des données complémentaires à la description originale de Richard: structure de l'antenne et de la branche externe de l'endite de la patte thoracique I, ont été apportées par Smirnov (op. cit.). La comparaison de ces mêmes structures montrent de nettes différences entre la forme australienne et sudaméricaine. Chez les spécimens du Vénézuéla, le ler article de l'endopode présente une soie fine, relativement courte (fig. 5), de sorte que la formule antennaire est en réalité : $O(1) .0(0) .3(1) / / 1(0)$. 1(0). 3(1).

Les 3 épines antennaires sont plus développées que figuré pour la forme australienne. Les 2 épines terminales ont au moins 1,5 fois la longueur de l'ar. ticle qui les porte et l'épine du $2^{\circ}$ article de l'exopode atteint la moitié de l'article distal (fig. 6). On observe, en outre, au niveau de l'endopode 2 rangées de longues spinules insérées l'une au $1 / 3$ proximal du ter article, l'autre dans la partie distale de l'article intermédiaire.
La branche externe de l'endite de P.I. (fig. 7-8) présente également 2 soies mais avec des sétules proximales moyennement développées de sorte que ces 2 soies ne sont pas véritablement transformées en crochet comme chez la forme australienne (cf. fig. 49 B. Smirnov, op. cit.).

Le rostre, obtus (fig. 9), n'apparaît pas franchement tronqué. 3 pores céphaliques principaux connectés (fig. $9,10,11$ ), proches du bord postérieur du bouclier céphalique (distance postpore inférieure à distance interpores) ont été observés. Les pores secondaires n'ont pas été vus. Il est difficile d'ap précier sur la fig. $49 \mathrm{~A}$ (Smirnov, op. cit.) si les 3 pores céphaliques sont, ou non, connectés, mais la distance PP est chez les spécimens australiens et vénézuéliens également inférieure à la distance IP. Chez nos individus, la taille relative des 3 pores céphaliques est apparue variable non seulement d'un individu à l'autre (fig. 9, 10, 11) mais encore chez un méme individu comme on peut le voir sur la figure 11 , relative à un spécimen en cours d'exuviation.

L'observation comparative montre que les 2 formes, australienne et vénézuélienne, paraissent ne pas être absolument similaires. En ce qui concerne certains caractères comme la structure des pores céphaliques, celle des appendices thoraciques ou même la spinulation antennaire, les travaux anciens ne nous fournissent pas de termes de comparaison, ces critères ne retenant pas, à cette époque, l'attention des auteurs. L'étude d'un matériel plus large, incluant des spécimens de diverses régions de l'Amérique du Sud, est nécessaire pour déterminer si la forme australienne et la forme sud-américaine constituent ou non un même taxon.

\section{Répartition}

En Amérique du Sud : Chili (Richard 1897) ; Pérou (Delachaux 1918); Brésil (Brehm 1937); Argentine (Olivier 1962); Vénézuéla (ce travail).

\section{Alona karelica Stenroos, 1897}

(PI. IX, fig. 1-11)

Matériel : 2 femelles parthénogénétiques.

LLO (E. Bl.), Litt. (Paspalum repens) $-370 \mu, 390 \mu$.

La présence dans le bassin de l'Orénoque de cette espèce, considérée comme une forme paléarctique, est inattendue. Il faut cependant rappeler que Alona karelica a été récernment signalée dans la zone orientale (Idris 1983 : Malaisie : Alona cf. karelica). Les 

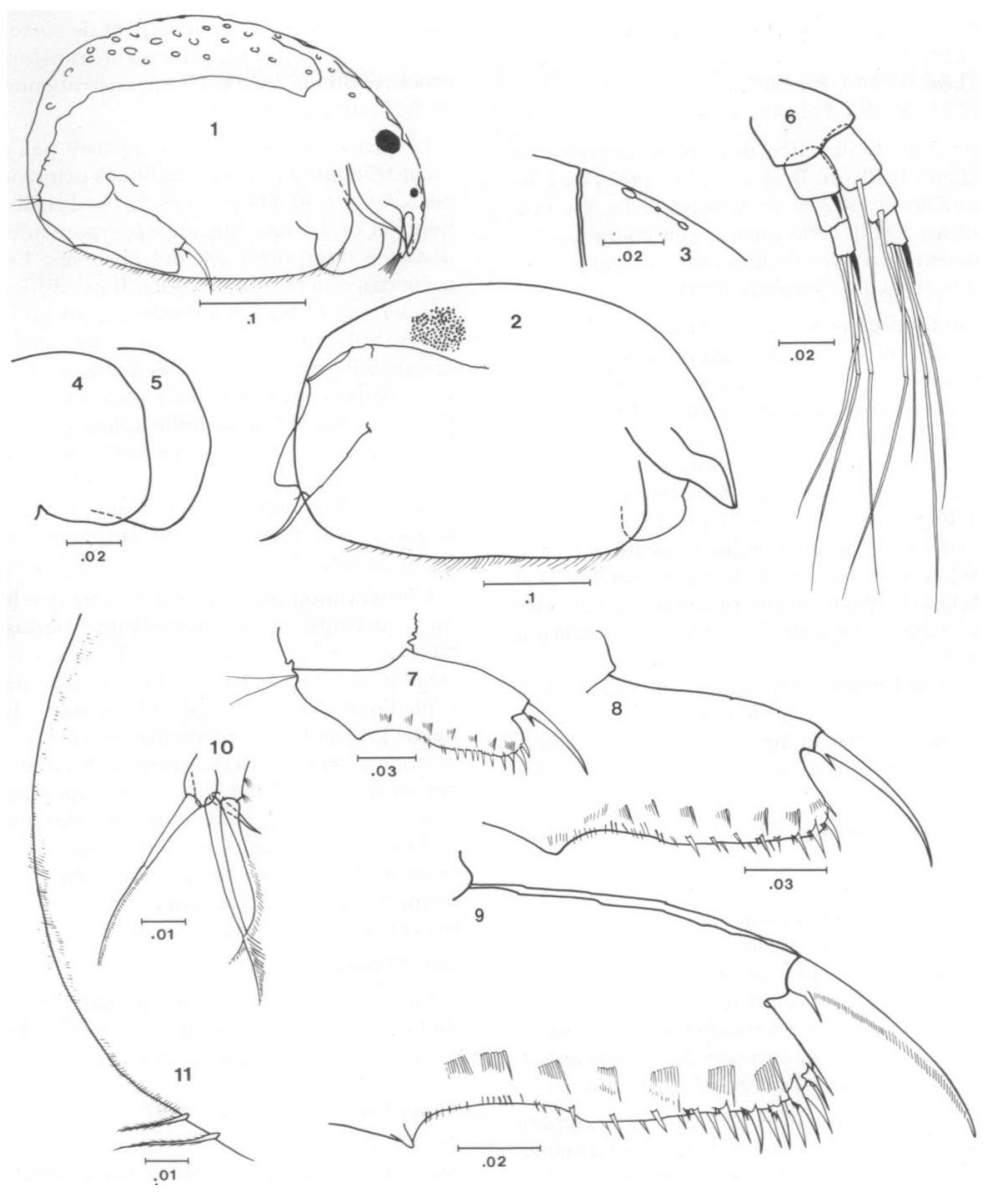

Planche IX

Fig. 1 à 11 : Alona karelica Stenroos, 1897 . Femelle parthénogénétique. 1 et $2:$ vue générale latérale ; $1:$ individu à valves tuberculées; 2 : individu à valves ponctuées. $3:$ pores céphaliques. 4 et $5:$ labre. $6:$ antenne. 7 à $9:$ postabdomen. 10 : exopodite et branche externe de l'endite de la patte thoracique 1.11 : angle postéro-ventral et bord postérieur de la valve gauche, face interne. 
2 femelles récoltées se rattachent à l'espèce karelica telle qu'elle a été redécrite et figurée du nord de l'Allemagne par Flössner (1963, 1972) et Herbst (1974).

- Le contour général de la carapace (fig. 1) : angles postéro-dorsal et postéro-ventral arrondis, bord ventral à peu près rectiligne, et celui du postabdomen (fig. 7-9) : bord dorsal et ventral parallèles, incisure profonde à la base de la griffe, lobe dorso-distal nettement saillant :

- La forme du labre (fig. 4-5) ; quadrangulaire, à apex arrondi ;

- La taille de l'ocelle (environ le quart de celle de l'œil):

- L'armature du postabdomen (fig. 8-9) : 9 à 10 denticules marginaux allongés et aigüs, les plus longs, serratés sur leur bord concave, situés à l'angle dorso-distal ; 2 à 3 groupes de courtes sétules marginales et submarginales au niveau de la dépression anale ; 6 fascicules latéraux de fines soies dont la plus distale est plus épaisse et plus longue, correspondent à la forme européenne figurée par Flössner (1972, fig. 146 A-F) et Herbst (1974, fig. 6-10).

La griffe terminale ( $f$ ig. 9), longue et fine, présente sur son bord concave, une délicate ciliature qui s'épaissit légèrement dans la moitié distale. L'épine basale est grêle et très courte.

L'antennule atteint l'apex du rostre (fig. 1). 2 des papilles sensorielles sont plus longues que les autres, comme figuré par Herbst (1974, fig. 8) pour la forme allemande.

Les 3 pores céphaliques principaux (fig. 3) sont connectés. Le pore intermédiaire est apparu plus petit que les 2 autres. La distance post-pore est légèrement inférieure à la distance inter-pores comme chez les individus de Malaisie (Idris 1983, fig. 52 D) mais les pores secondaires figurés par cet auteur n'ont pas été vus.

Les antennes (fig. 6) sont moyennement longues.

L'article proximal de l'endopode porte une soie assez fine, plus courte que les autres soies antennaires.

Formule antennaire : $0(1) .0(0) .3(1) . / 1(0) .1(0) .3(1)$.

Les 3 épines antennaires sont relativement bien développées. Epines terminales chacune aussi longue que le segment qui la porte ; épine du ler article de l'endopode presque aussi longue que le seg. ment intermédiaire.
La branche externe de l'endite de la patte thoracique $I$ (fig. 10) porte 3 soies. Soie 1 courte, en crochet. Soies 2 et 3 longues et grêles. La soie in termédiaire un peu moins longue est finement sétulée sur un peu plus de sa moitié distale, la soie 3 porte des sétules de longueur décroissante dans le tiers distal seulement.

La soie de l'exopodite, environ de mème longueur que la soie 3 , est finement ciliée dans sa moitié distale.

Les 2 femelles étudiées montrent une sculpture de la carapace différente : tuberculée chez l'une ( $f$ ig. 1), seulement finement ponctuée chez l'autre (fig. 2). Tous leurs autres caractères morphologiques sont similaires. Les soies du bord ventral des valves, relativement longues dans la région antérieure et dans la moitié postérieure des valves, sont séparées par un groupe de soies plus courtes. Toutes les soies sont bisétulées. Elles sont suivies par une rangée de très fines sétules qui dépassent largement le bord des valves au niveau de l'angle postéro-ventral (disposition rare dans le genre Alona) puis deviennent progressivement submarginales en direction dorsale (fig. 11).

\section{Répartition}

La récolte d'Alona karelica dans la zone néotropicale jointe à l'observation d'Idris (1983) pour la région orientale modifient considérablement les données biogéographiques relatives à cet te espèce. Alona karelica n'avait jamais été signalée sur le continent américain.

\section{Biapertura intermedia (Sars, 1862)}

(Pl. X, fig. 1-6)

LLO (E. Bl.), Litt. (Eichhornia crassipes) - $335 \mu$.

Curieusement, un seul exemplaire de cette forme cosmopolite, assez largement rencontrée, a été récolté.

La forme générale du corps, du labre et du postabdomen (fig. 1) ne montrent pas de caractéristiques particulières.

L'armature du postabdomen (fig. 5-6) (10 denticules marginaux courts et triangulaires, serratés sur leur face concave ; 9 fascicules latéraux de soies spiniformes dont la distale, plus robuste et très longue, dépasse presque les denticules marginaux dans l'angle postéro-dorsal) correspond bien à la forme type. 

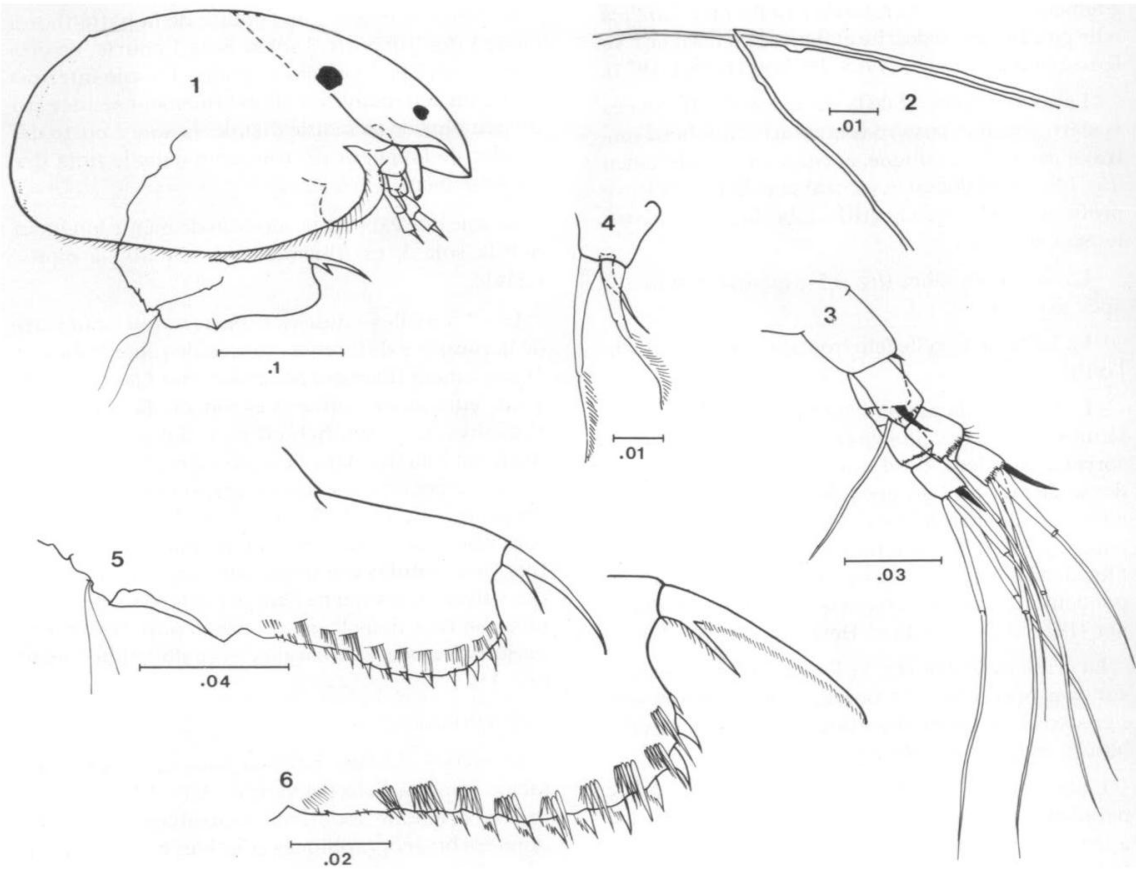

Planche $\mathbf{X}$

Fig. 1 à 6 ; Biapertura intermedia (Sars 1862). Femelle parthénogénét ique. 1 ; vue générale latérale. 2 : pores céphaliques. 3: antenne. 4 : branche externe de l'endite de la patte thoracique 1 . 5 et $6:$ postabdomen.

La soie du ler article de l'endopode est fine et courte (fig. 3). L'épine terminale de l'endopode est oresque 2 fois plus courte que l'épine distale de l'exopode. L'épine du ler article de l'exopode est bien développée. Le basipode porte une toute petite épine dans la région distale, sur la face externe, au voisinage des 2 branches antennaires.

Formule antennaire : $Q(1), 0(0) .3(1) . / 1(0) .1(0) .3(1)$.

La branche externe de l'endite (fig. 4) porte 3 soies : soie 1 courte, en crochet, soies 2 et 3 moyennement fortes, la soie intermédiaire un peu moins longue, toutes deux sétulées sur leur partie distale.

\section{Répartition}

En Amérique du Sud : Brésíl (Sars 1901, Brehm 1937) : Paraguay (Daday 1905, Stingelin 1906) : Pérou (Delachaux 1918); Vénézuéla (ce travail).

\section{Oxyurella ciliata Bergamin, 1939}

(Pl. XI, fig. 1-8)

Matérial : 3 femelles parthénogénétiques.

I \& : LJO (E. Bl.), Litt. (Eich homia crassipes) - $335 \mu$.

2 ९ : LLC (E. Bl.), Litt. (E. crassipes) $-420 \mu-430 \mu$.

Originellement décrite du Brésil par Bergamin (1939), cette espèce caractérisée par son labre 


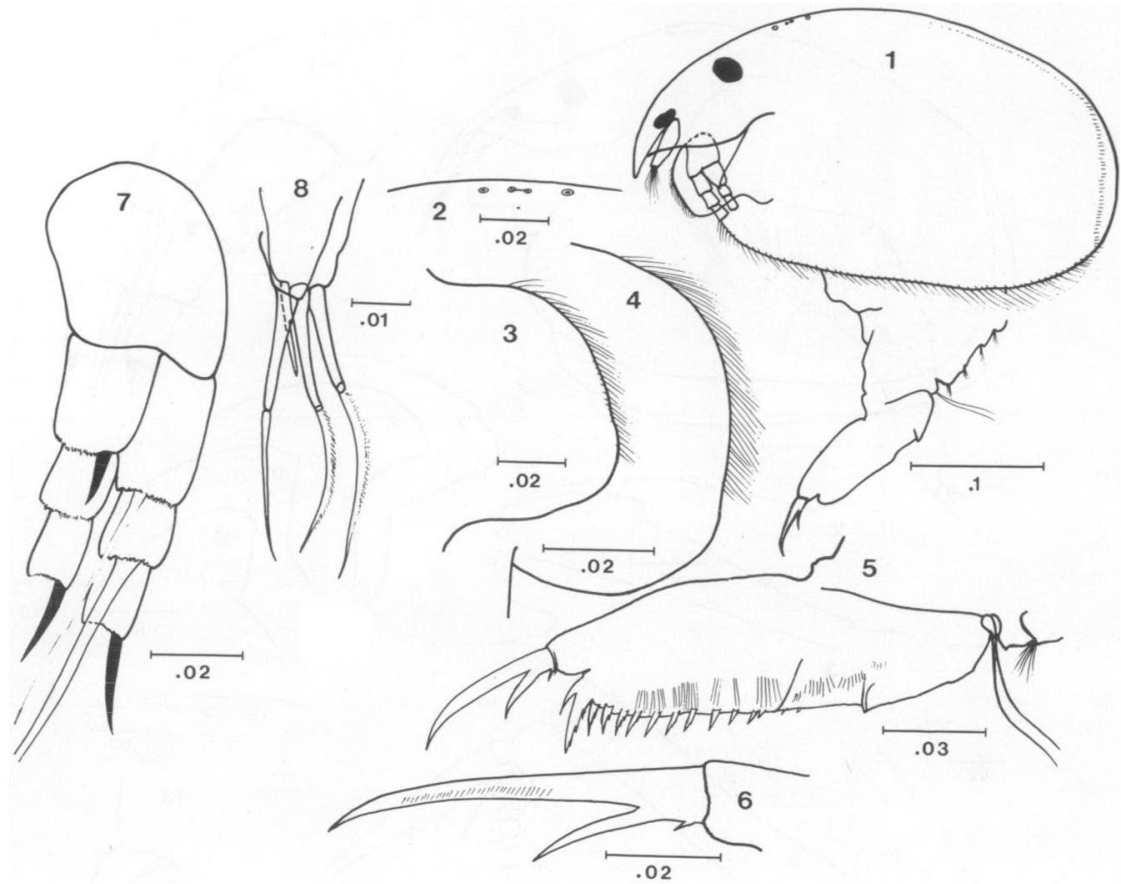

Planche XI

Fig. 1 à 8 : Oxyurella ciliata Bergamin 1939. Femelle parthénogénétique. 1 : vue générale latérale. 2 : pores céphaliques. 3 et 4 : labre. $5:$ postabdomen. $6:$ griffe postabdominale. $7:$ antenne (soies terminales non figurées). $8:$ exopodite et branche externe de l'endite de la patte thoracique I.

pubescent n'a, depuis sa description, été retrouvée qu'une seule fois et en 1 seul exemplaire. du lac Peten, Guatemala (Van de Velde \& al. 1978).

La femelle du lac Peten montre une pubscence qui s'étend sur tout le bord antérieur et sur une partie du bord ventral du labre ( $f i g$. 2b, Van de Velde \& al. op. cit.). Chez les spécimens vénézuéliens, la pubescence s'arrête bien avant l'apex. Toute la partie distale du labre, largement arrondie, apparaît totalement glabre (fig. 34). Les autres caractères : contour de la carapace, sétation du bord ventral et postérieur des valves (fig. 1), structure des pores céphaliques $(f i g$. 2) ( 2 pores principaux séparés par 2 pores intermédiaires connectés avec, de chaque côté, 1 petit pore secondaire), forme et armature du postabdomen (fig. 5) (11 à 12 denticules marginaux serratés sur leur face concave, 4 peignes latéraux subrectangulaires de soies très fines) sont similaires à la forme guatémaltèque.

La griffe terminale finement ciliée porte une épine basale relativement longue, légèrement sigmoïde chez nos individus (fig. 6) et une courte spinule proximale. 


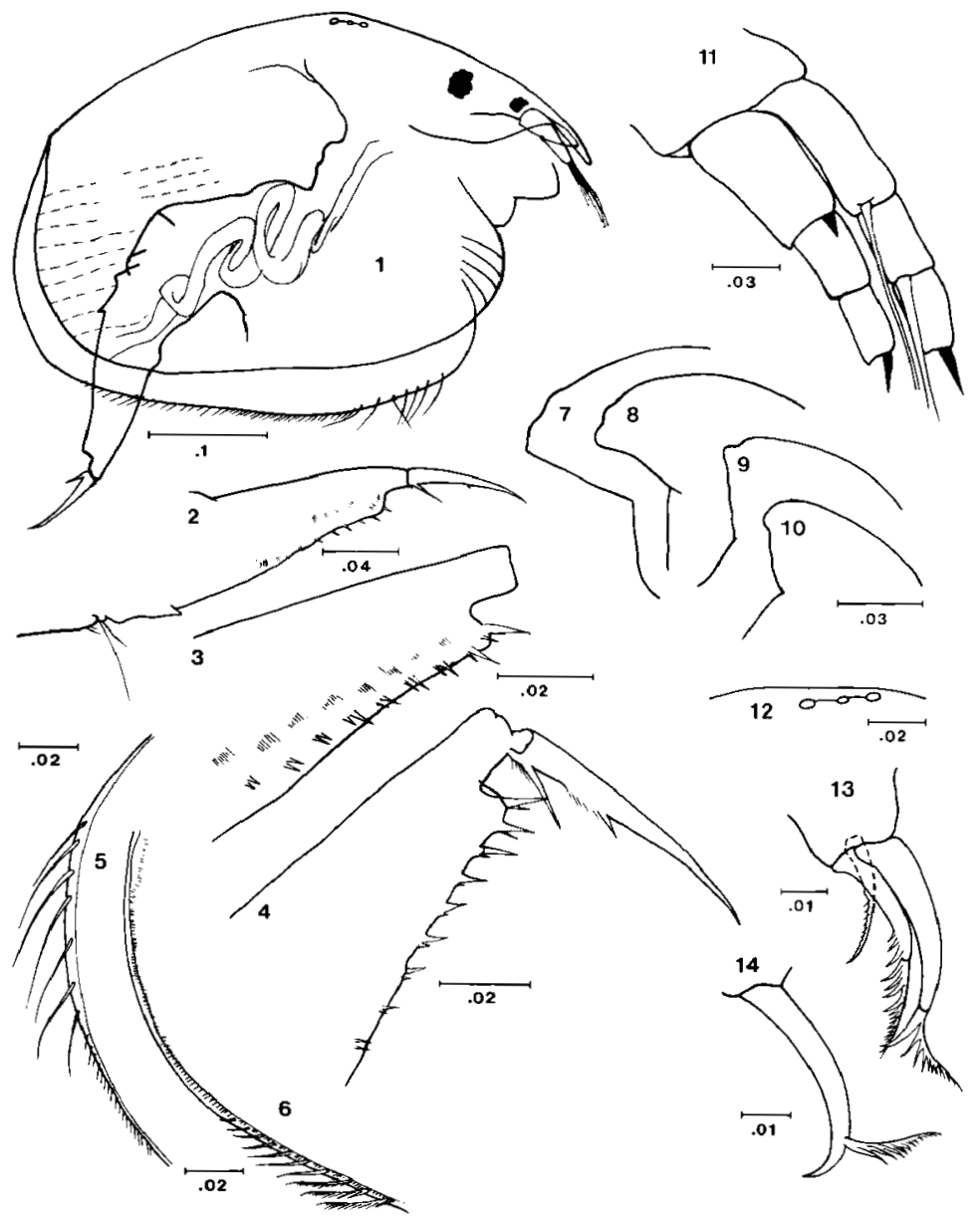

Planche XII

Fig. 1 à 14 : Kurzia latissima (Kurz 1875). Femelle parthénogénétique. 1 : vue générale latérale. 2 à 4 : postabdomen. 5 : région antéro-ventrale de la valve droite, face interne. 6 : angle postéro-ventral de la valve gauche, face interne. 7 à $10:$ labre. 11 : antenne (soies terminales non figurées). 12 : pores céphaliques principaux. $13:$ branche externe de l'endite de l'appendice thoracique 1.14 : soie 3 en crochet, avec son flagelle sétulé. 
La partie distale des articles antennaires est très délicatement denticulée (fig. 7). Les 3 épines antennaires sont bien développées.

Formule antennaire : $Q(1) .0(0) .3(1) . / 1(0)$. 1(0). 3(1).

La branche externe de l'endite (fig. 8) porte 3 soies : soie 1 , courte, en crochet, soies 2 et 3 longues et grêles, subégales, toutes trois courtement sétulées sur un rxu plus de leur moitié distale.

La soie de l'exopodite, également longue et grêle est très finement ciliée.

Répartition

En Amérique du Sud : Brésil (Bergamin 1939, 1941) :

Vénézuéla (ce travail).

\section{Kurzia latissima (Kurz, 1875)}

(PI. XII, fig. 1-14)

Matériel: 8 femelles parthénogénétiques.

6 १ ९ : LRC (E. Bl.), Pél - $335 \mu$ à $430 \mu$.

2 ९ Q: RUP (E. Bl.), Pél - $415 \mu, 465 \mu$.

Mentionnée plusieurs fois en Amérique du Sud au début du siècle (Brésil-Argentine : Sars 1901, Paraguay : Daday 1905, Colombie: Delachaux 1913) Kurzia latissima n'a curieusement plus été signalée depuis, à la différence de l'autre espèce du genre $(K$. longirostris) dont elle diffère nettement par la longueur du rostre, le contour du labre, la forme et l'armature du rxostabdomen.

Les individus vénézuéliens sont très similaires à la forme européenne figurée par Lilljeborg (1900), Flössner (1972). L'armature du postabdomen est représentée par 8 à 9 denticules, marginaux grêles et aigus, de longueur croissante distalement, chacun d'eux accompagné d'un second denticule de petite taille (fig. 2-4). La griffe terminale (fig. 4) porte dans sa moitié proximale et sur son bord concave une rangée de fines soies dont la dernière, située à moitié griffe environ, forme un petit denticule. L'épine basale de la griffe est également finement sétulée dans sa partie proximale.

Le contour de la carapace (fig. 1) correspond à celui de la forme typique. Les valves finement ponctuées dans la population du lac Rio Claro, présentent 16 à 20 stries longitudinales chez les exemplaires du Rio Upata.

La sétation du bord ventral des valves, moins uniforme que figurée par les auteurs, se répartit en 3 groupes de soies (fig. 1, 5, 6) : 6 à 7 soies antéroventrales longues et largement espacées, 24 à 25 soies courtes et rapprochées atteignant la moitié de la marge ventrale et un $3^{\circ}$ groupe de soies de longueur intermediaire qui se raccourcissent distalement. Une rangée submarginale de fines sétules arme la face interne de l'angle postéro-ventral et du bord postérieur des valves (fig. 6).

L'apex du labre (fig. 7-10), habituellement lisse et légèrement arrondi, apparaît chez presque tous les individus plus ou moins tronqué, avec 1 ou 2 crénulations, rappelant par ce caractère la forme australienne figurée par Smirnov (1983, fig. 61 A).

L'antennule longue et grêle porte de 7 à 8 papilles sensorielles dont 3, plus développées, sont de longueur subégale à l'antennule.

L'épine du ler segment de l'exopode antennaire est courte et large comme figuré par Lilljeborg (1900, Tab. LXVI, fig. 3) ; par contre, les 2 épines terminales antennaires apparaissent 2 à 3 fois plus longues que celles de la forme européenne. Le ler segment de l'endopode porte 1 soie fine et plus courte que les autres (fig. 11).

Formule antennaire : $0(1) .0(0) .3(1) / 1(0) .1(0) .3(1)$.

La branche externe de l'endite de la patte thoracique I, connue pour Kurzia longirostris (Smirnov 1971, fig. $116 \mathrm{C}$ ), montre une structure analogue : 3 soies de longueur inégale : soie 1 , courte, en crochet, soie 2 et 3. plus longues et épaisses (fig. 13). La soie intermédiaire est garnie de sétules sur un peu plus de la moitié de sa partie distale. La soie 3 est transformée en crochet par développernent de la sétule proximale prolongeant la partie basilaire, les autres sétules constituant une sorte de flagelle armé de sétules de taille décroissante distalement (fig. 14).

Répartition

En Amérique du Sud : Brésil, Argentine (Sars 1901); Paraguay (Daday 1905) ; Colombie (Stingelin 1913) ; Vénézuéla (ce travail).

\section{Acroperus cf. harpae (Baird, 1834)}

(PI. XIII, fig. 1-23)

Matériel : 7 femelles parthénogénétiques dont 2 femelles ovigères.

RUR (E. No.), Litt. (Eichhomia crassipes) - $515 \mu$ à $615 \mu$.

Femelles ovigères : taille minimale observée : $515 \mu$.

Le genre Acroperus est pauvrement représenté en Amérique du Sud. Les seules mentions sont celles de Acroperus harpae au Paraguay (Daday 1905: 


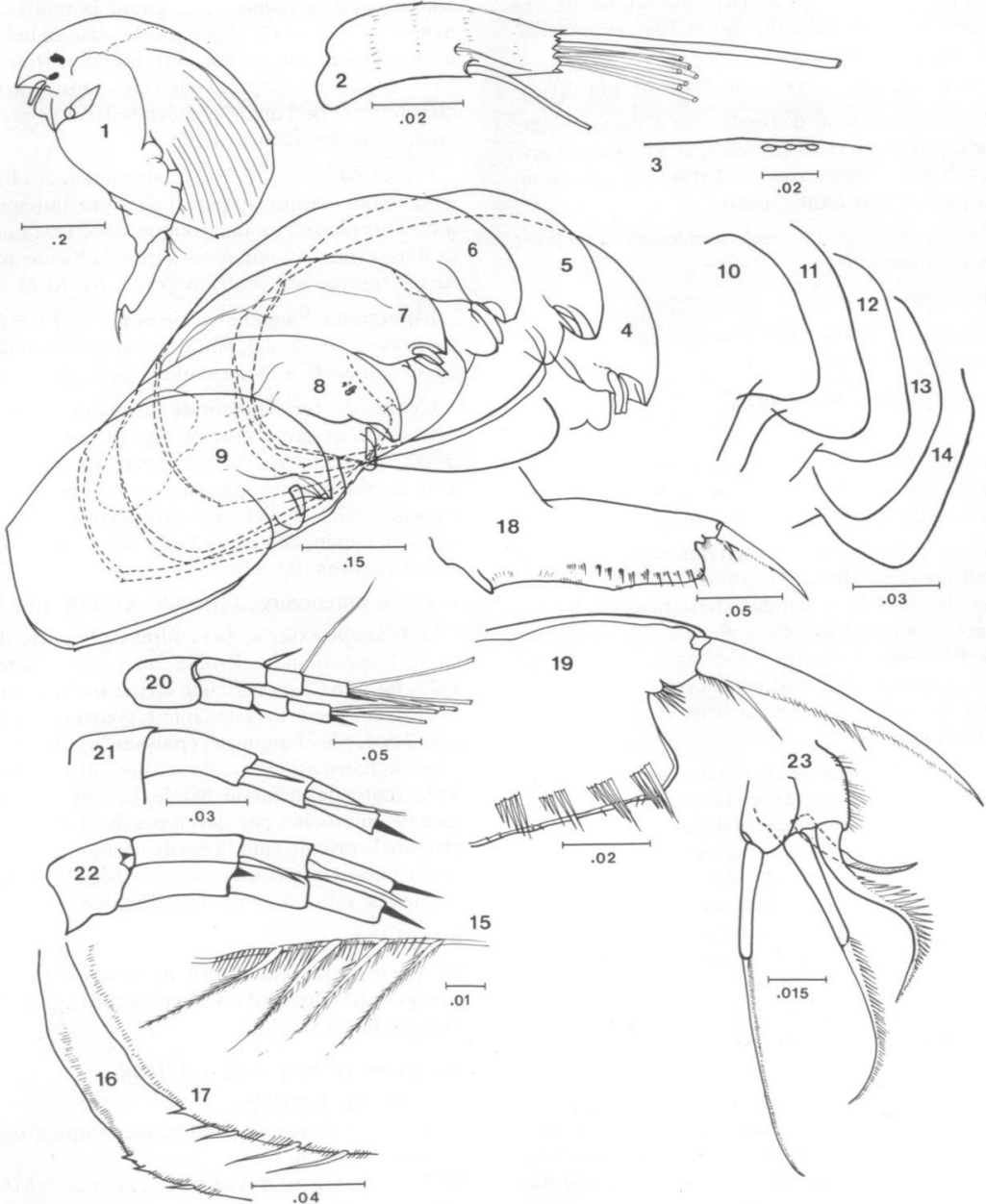

Planche XIII

Fig. 1 a $23:$ Acroperus of harpae (Baird 1834). Femelle parthénogénétique. 1 : vue générale latérale. $2:$ antennule. 3 : pores céphaliques, 4 à 9 : contour général de la carapace. 10 à 14 : labre. 15 : sétulation du bord ventral des valves. 16 et $17:$ angle postéro-ventral, valve gauche, face interne, de 2 spécimens. 18 : postabdomen. $19:$ partie terminale du postabdornen. 20 à 22 : antenne (20: segment distal des soies terminales non figuré ; 21 et $22:$ soies terminales non figu. rées). 23 : exopodite et branche externe de l'endite de la patte thoracique $I$. 
simple citation sans figures) et au Brésil (Thomasson 1954), et celle de Acroperus titi (Harding 1955), decrite originellement du bassin du lac Titicaca comme Alonopsis titi.

Tous les exemplaires vénézuéliens montrent une carène céphalique bien développée (fig. 1). Le rostre est court et apparaît parfois tronqué à son extrénité. La hauteur maximale de la carapace varie de $62 \%$ à $66 \%$ de la longueur et se situe dans la moitié antérieure du corps; la hauteur du bord postérieur libre des valves représente $62 \%$ à $70 \%$ de la hauteur maximale. Les antennules se projettent généralement audelà du rostre (fig. 1, 4-9).

Labre court, à bord antérieur légèrement convexe et apex largement arrondi (fig. 10-14).

Soies du bord ventral de la carapace courtement bisétulées et séparées par des groupes de 4 à 5 longues spinules insérées submarginalement sur la face interne des valves (fig. 15).

Angle postéro-ventral avec un, parfois deux, très netits denticules, suivis par une rangée submarginale de courtes sétules sur le bord postérieur des valves (fig. 16-17).

Denticules marginaux du postabdomen réduits en taille et en nombre, peu visibles à faible grossissement (fig. 18). 11 a 12 fascicules latéraux de soies spiniformes dont la plus distale est la plus développée; les 4 à 5 fascicules distals dépassent largement le bord dorsal du postabdomen. Griffe terminale longue et mince, finement ciliée dans sa partie distale, sétulée dans sa moitié proximale, la dernière sétule formant un petit denticule. L'épine basale, grêle, porte une délicate ciliature de taille croissante distalement.

Le lobe distal de l'échancrure du postabdomen est armé de 6 à 7 courtes soies rayonnantes (fig. 19).

Antennes rẹlativęmęnt peu développées.

La soie du ler article de l'endopode, parfois interDrétée comme une épine (Smirnov 1971. Negrea 1983), est de longueur subégale au 2 ème article (fig. 20-21). L'épine du ler segment de l'exopode est très courte (fig. 22). Le basipode porte dans la région médio-distale et sur sa face exteme une toute petite épine.

Formule antennaire: $0(1)$. $0(0)$. 3(1)/1(0). 1(0). 3(1).

La branche exteme de l'endite (fig. 23) porte 3 soies : soie 1 , courte, en crochet; soies 2 et 3 longues, relativement épaisses; soie 2 sétulée sur les $2 / 3$ distals, soie 3 plus courtement sétulée sur un peu plus de sa moitié distale.

La soie de l'exopodite, plus longue et grêle, est finement ciliée.

Par leur antennule se projetant nettement au-delà du rostre les spécimens vénézuéliens se rapportent à l'espèce australienne Acropertis sinuatus, mais les valves de cette dernière espèce sont dépourvues de tout denticule à l'angle postéro-ventral et leur bord posterieur est sinueux " convex about the middle, concave dorsally »(Henry 1919, p. 471).

Ils ne sont pas, non plus, exactement conformes à la forme typique dont ils different par:

- le labre, court, arrondi, et non allongé, triangulaire, à apex plus ou moins aigu comme figuré par Lilljeborg (1900, Tab. LXXII, fig. 16), Flössner (1972, Tab. 134, fig. A) et Smimov (1971, fig. 483);

- la soie spiniforme du ler article de l'endopode, 2 fois plus longue qu'elle n'apparaît chez. la forme paléarctique (Lilljeborg 1900, Tab. LXIII, fig. 17, Smirnov 1971, fig. 483).

- le rostre court, ne recouvrant pas les antennules.

Néanmoins, la structure du postabdomen, de la griffe terminale, de même que celle des soies de la branche externe de l'endite de la $1^{\circ}$ patte thoracique sont très proches de la forme typique ; c'est pourquoi nous rapportons ces exemplaires, quoique avec réserve, à Acroperus harpae.

\section{Répartition}

En Amérique du Sud : Paraguay (Daday 1905); Brésil (Thomasson 1954); Vénézuéla (ce travail).

\section{6. - Discussion}

Si l'on établit le bilan faunistique de ce travail, on constate que l'étude de 16 corps d'eaux (12 milieux lénitiques, 4 milieux lotiques) répartis dans le bassin moyen de l'Orénoque, a permis d'identifier 58 taxa dont 24 (23 espèces et 1 sous-espèce) sont nouveaux pour le Vénézuéla. 2 espèces, Alona ovata et Bosminopsis macaguensis, sont nouvelles pour la science. Alona karelica, élément d'origine paléarctique, est mentionnée pour la première fois sur le continent américain. Dunhevedia crassa et Alonella hamulata sont nouvellement citées pour l'Amérique du Sud.

En outre, le matériel échantillonné a fourni plusieurs individus de Leydigiopsis ormata Daday dont 
Tableau II. Répartition des espèces aux différentes dates d'échantillonnage : échantillons littoraux.

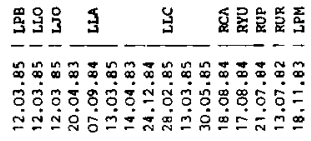

P. ramosa

L. australis

S. sernulatus

H. spinosa

E. trisetialis

1. spinifer

G. bhazzai

D. dadayi

D. odontoplax

D. mach ops

A. excisa

A. brasiliensis

A. hamulata

E. tridentatus

E. hubridas

C. nitidulus

C. pubescens

C. Curynotus

A. quttata

A. qlobulosa

A. quadrangularis

A. eximia

B. vernucasa

B. affinis

B. karua

C. dadayi

o. ciliata

K. Rongirostris

G. testudinatia

L. curvinostris

L. ornata

A. Co harpae
A. rustica
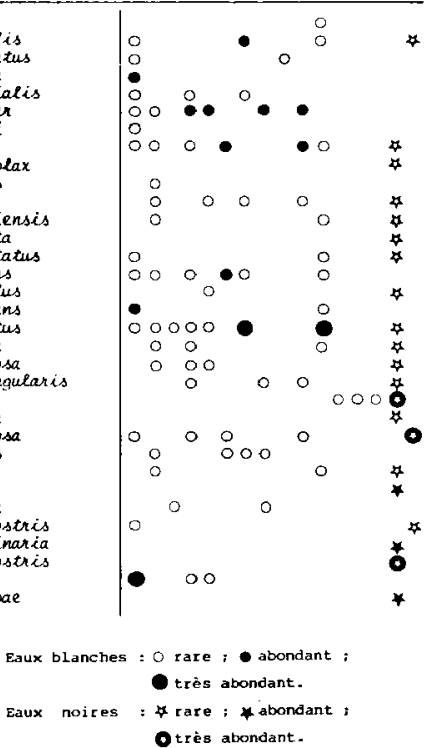

un mâle et une femelle éphippiale, inconnus iusqu'ici, qui sont décrits séparément (Rey \& Vàs. quez 1986).

Le recensement des données de la littérature con. cernant la faune cladocérienne du Vénézuéla et cel. les du récent travail effectué sur les savanes inondables de Mantecal, état Apure (Zoppi de Roa, sous oresse), indiquent un total de 66 taxa valides. Les différents corps d'eaux étudiés dans le bassin moyen de l'Orénoque portent à 90 le nombre total d'espèces de Cladocères actuellement connues pour la faune vénézuélienne.
Si l'on considère l'aspect biogéographique, il apparaît que l'essentiel de la faune récoltée est, bien sûr, d'origine tropicale. $16 \%$ seulement des especes identifiées sont cosmopolites. $35 \%$ du peuplement sont des formes néotropicales à plus ou moins large répartition, parmi lesquelles 8 sont des endémiques sud-américaines: Pseudosida ramosa, Diaphanosoma spinulosum, Diaphanosoma brevireme, Daphnia gessneri, Alonella brasiliensis, Ephemeroporus tridentatus, Camptocercus dadayi, Leydigiopsis curvirostris. Le reste de la faune est constitué en majorité de formes pantropicales ou subtropicales auxquelles s'ajoutent quelques espèces à répartition moins tranchée, telles Alona rustica, Kurzia latissima, distribuées dans l'hémisphère nord et la région néotropicale, et Alona globulosal, espèce circumtropicale dont l'aire de répartition englobe le continent nord-américain. Quant à Alona karelica et Alona guttata tuberculata, toutes deux d'origine paléarctique, leur distribution en Europe-USSR d'une part, et sur le continent sud-américain d'autre part (Vénézuéla et Colombie respectivement), montre une nette disjonction.

La distribution des espèces de Cladocères dans les divers corps d'eaux étudiés est schématisée dans les tableaux II (échantillons littoraux) et III (échantillons pélagiques).

Ces tableaux rendent compte de la présence et $\mathbf{d u}$ degré d'abondance des espèces, aux différentes dates d'échantillonnage. Un figuré différent est utilisé pour distinguer les 2 catégories de milieux : eaux blanches, eaux noires. Dans ces tableaux ont été omis les relevés pour lesquels aucun indivigu n'a été observé.

Du point de vue de l'évolution saisonnière de l'abondance des espèces, seuls les relevés pélagiques permettent d'établir une relation avec le cycle climatique. Au vu du tableau III et de la liste générale des dates d'échantillonnage, il apparaît que les espèces montrent une grande abondance pendant la saison des plujes (mai, juin-novembre, décembre), avec généralement une plus grande abondance de mai à août, début de la saison chaude et pluvieuse, observation qui rejoint celles de Zoppi de Roa (1972) dans la " laguna " de Campoma (Vénézuéla). Les relevés

1. Alona globulasa fait actuellement l'objet d'une révision et d'un reclassement dans un nouveau genre (Pr. C.H. Fernando, in litt.) 
Tableau III. Répartition des espèces aux différentes dates d'échantillonnage : échantillons pélagiques.

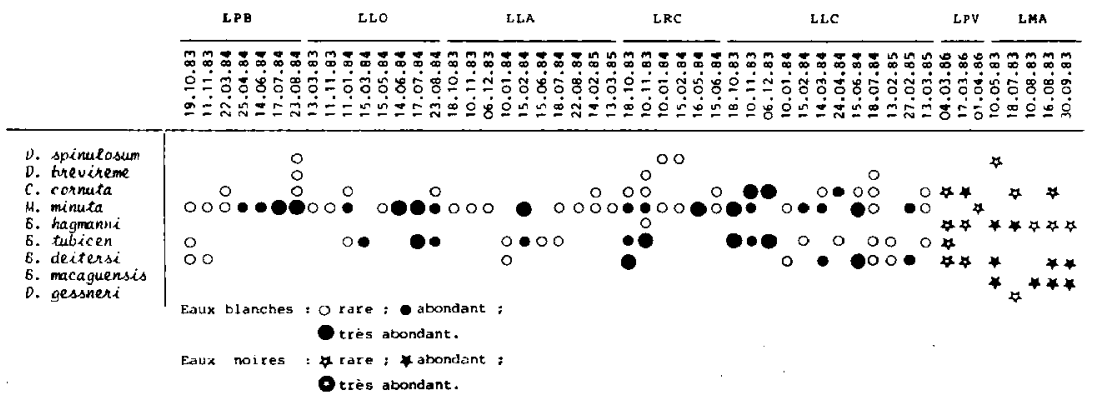

effectués en saison sèche (cf. ch. 3.1.1.) n'ont très souvent livré aucun ou très peu d'individus.

Du point de vue diversité spécifique, la faune la plus riche de Cladocères (14 à 23 espèces selon les sites) a été observée au niveau des lacs d'inondation de l'Orénoque, la contribution la plus importante à cette diversité étant due aux espèces littorales Tableaux II et IV). La plus grande diversité spécifique, enregistrée à Los Castillos, s'explique probablement en partie par l'analyse d'un nombre comvarativement plus élevé de relevés, tant pélagiques que littoraux.

Pour le Rio Uracoa (eaux noires), l'examen d'un seul échantillon littoral a révélé un total de 18 espèces, ce qui montre la richesse de cet habitat.

D'une manière générale, le peuplement en Cladocères de la zone littorale est constitué à $90 \%$ de Chydoridae. Le Rio Uracoa a montré le nombre le olus élevé d'espèces de cette famille : 18 Chydoridae sur les 18 espèces reçensées, suivi par les lacs d'inondation de l'Orénoque : Los Castillos avec 17 espèces, Playa Blanca avec 14 espèces.

Les formes littorales qui ont été les plus fréquemment rencontrées sont Chydorus eurynotus, Disparalona dadayi, Ephemeroporus hybridus, Alonella excisa, Biapertura verrucosa.

Les stations échantillonnées dans les Rios Camoiràn, Yuruani et Upata (eaux blanches), rangées dans un souci de simplification avec les relevés effectués en zone littorale (Tableau II), correspondent en fait à un milieu lotique différent des autres biotopes littoraux. Ce caractère se retrouve au niveau du peuplement, pauvrement représenté sur le plan spécifique, mais qualitativement intéressant par la présence d'Alona eximia. La récolte de cette espèce en pleine eau dans les trois rios, montre sa préférence pour les milieux lotiques et confirme les observations de Kiser 1948 (Pearl River, Chine), Green 1962 (Sokoto River, Afrique de l'Ouest), Chien 1970 (Pearl River, Etats-Unis) et Paggi 1979 (Parana River, Argentine).

Toutefois, cet habitat ne doit pas représenter une exigence stricte pour Alona eximia, car Green lop. cit.) signale aussi sa présence dans un petit étang isolé par la décrue, de mème que nous l'avons obser. vée, abondante, dans le biotope littoral à Eichhornia échantillonné dans le Rio Uracoa.

En ce qui concerne le peuplement pélagique, le nombre d'espèces limnétiques enregis trées est relativement bas, caractéristique connue du peuplement zooplanctonique en climat tempéré mais également rapportée pour les régions tropicales (Fernando 1980).

Les milieux pélagiques étudiés ont montré les espèces limnétiques suivantes: Moina minuta, Bosminopsis deitersi, Ceriodaphnia comuta, Bosmina tubicen, Bosmina hagmanni. Diaphanosoma spintt. losum, Diaphanosoma brevireme. Cette composition fałınistique est similaire à celle des lacs d'inondation de l'Amazone (Hardy \& al 1984, Robertson \& Hardy 1984). 
Tableau IV. Nombre total d'espèces recensées dans 6 corps d'eaux du systeme Orénoque-Caroni et dans une rivière d'eaux noires (RUR).

Entre parenthèses : nombre d'espèces pélagiques et lit. torales respectivement. $P$ : espèces pélagiques uniquement. $\mathrm{L}$ : especces littorales uniquement.

\begin{tabular}{cccccc}
\hline LPB LLO L.LA LLC & LMA & LPV & RUR \\
$21(7,14) 14(3,11) 17(4,13) 23(6,17)$ & $6(P)$ & $5(P)$ & $18(L)$ \\
\hline
\end{tabular}

Si l'on compare les milieux des eaux blanches (lacs d'inondation de l'Orénoque) et ceux des eaux noires (lac de barrage de Macagua), on observe l'association M. minuta, C. cornuta, B. tubicien, B. deitersi, $D$. spinulosum dans la première catégorie, et l'association B. hagmanni, B. deitersi, Bosminopsis macaguensis, $C$. cornuta, $D$. spinulosum dans la deuxième.

Outre un groupe d'espèces communes aux 2 types de milieux (C. comuta, B. deitersi, D. spinulosum), il apparaît que Moina minuta, forme dominante dans les lacs d'inondation est absente dans le lac de Macagua, alors que Bosminopsis macaguensis, absent dans les lacs d'inondation, est régulièrement présent dans les relevés du lac de barrage.

Dans une étude comparative des lacs d'inondation des eaux blanches et noires du système Amazone, Brandorff (1978) a trouvé un nombre plus élevé d'espèces de Bosminidae dans les eaux noires, à la différence des eaux blanches où prédominaient les espèces de Daphniidae. Heredia-Seixas (1981), travaillant sur différents corps d'eaux du Brésil, rapporte que les Bosminidae tendent à dominer dans les milieux à basses valeurs de $\mathrm{pH}$ et de conductivité, tandis que les espèces de Daphniidae tendent à être dominantes dans les eaux à $\mathrm{pH}$ et conductivité plus élevés.

Dans notre cas, les résultats obtenus pour le lac de Macagua (dominance nette des espèces de Bosminidae dans les eaux noires à faibles valeurs de $\mathrm{pH}$ et de conductivité) sont en accord avec ceux obtenus dans les études ci-avant mentionnées.

Il n'en est pas de mème pour le peuplement pélagique des lacs d'inondation de l'Orénoque dans lesquels la forme dominante est Moina minuta, les Daphniidae étant essentiellement représentés par Ceriodaphnia comuta. Daphnia gessneri, bien développée dans le Lago Castanho (eaux blanches)
(Brandorff 1978), également présente dans plusieurs autres corps d'eaux brésiliens (Zago 1978, Carvalho 1984, Robertson \& Hardy 1984, Sendacz 1984), n'a été observée que dans un seul relevé, provenant du lac de barrage sur le Caroni. Il est à noter que dans une étude extensive de différents corps d'eaux du Vénézuéla (environ 3000 échantillons provenant de 19 lacs et réservoirs), Infante (1984) a, de même, observé cette espèce uniquement dans un seul site, le lac de barrage de Guri, également localisé sur le Caroni mais dans la section supérieure du fleuve.

La rareté dans les eaux vénézuéliennes de cette forme endémique du système Amazone-Orénoque (Infante $o p$. cit.) est asse? surprenante, bien que les données de la littérature puissent peut-être expliquer cette situation.

Ainsi Carvalho (1984), ayant constaté la disparition de Daphnia gessneri dans le Lago Grande : lac d'inondation du fleuve Solimốes (Amazone), relie cette disparition à la forte prédation exercée sur cette espèce et aussi à la faible transparence du milieu en période de basses eaux. Toutefois, Brandorff (1977) ayant observé les plus fortes densités de $D$. gessneri durant cette même période, Carvalho explique ce fait par les valeurs de la transparence de l'eau, supérieures dans le Lago Castanho à celles du Lago Grande.

Par ailleurs, plusieurs auteurs établissent une relation entre la présence de $D$. gessneri et le degré de trophie du milieu. Ainsi, dans le lac Americana (Brésil), D. gessneri est progressivement remplacée par Diaphanosoma sp. avec l'eut rophisation croissante du milieu (Zago 1976). De même, dans le lac Billings (Brésil), réservoir en état avancé d'eutrophisation, on note la fréquence et l'abondance de Dia. phanosoma sp. alors que $D$. gessneri est faiblement développée (Sendacz 1984).

Des observations de ces différents auteurs, il ressort que la turbidité, la prédation et le degré d'eutrophisation sont des facteurs susceptibles d'in. fluencer directement ou indirectement la répartition de D. gessneri.

En ce qui concerne les milieux prospectés, la présence de $D$. gessneri dans les lacs de barrage de Guri et de Macagua pourrait être liée au caractère oligotrophe et à la haute transparence des eaux noires du Caroni.

Selon la mème hypothèse, son absence dans les lacs d'inondation de l'Orénoque s'expliquerait par 
a forte turbidité de ces milieux (Vàsquez \& Sanchez 1984, Vàsquez 1984) et par des conditions trophiques de l'environnement probablement plus élevées.

Dans les limites de nos observations, il est difficile d'aller au-delà de ces hypothèses. Il reste que, dans les eaux vénézuéliennes, cette espèce se révèle un élément absent (eaux blanches) ou très rare (eaux noires) dans les associations de Cladocères enregistrées.

\section{Remerclements}

Nous remercions le Dr. E. Zoppi de Roa (Caracas) qui a bien voulu nous communiquer le manuscrit de son travail sur les Cladocères des savanes inondables de Mantecal.

\section{Travaux cltés}

Bergamin (F.) 1935. Una nova especie de Cladocera encontrada na Directoria de Industria Animal. Rev. Industr. Animal., II (3): 284-285.

Bergamin (F.) 1939a. Os Cladocera. Rev. Industr. Animal., N.S, 2 (2): $80-86$.

Bergamin (F.) 1939b. Os Cladocera. Rev. Industr. Animal., N.S., 2 (4) : 87.92.

Bergamin (F.) 1941. Os Cladocera. Bol. Industr. Animal, 4 (1): 89.166

Birge (E.-A.) 1878. Notes on Cladocera. Trans. Wis. Acad. Sci., 4 : 77.110.

Birge (E.-A.) 1910. Notes on Cladocera. IV. Trans Wis. Acad. Sci., 16, part II (3) : 1017-1066

Brandoff (G.-O) 1977. Untersuchungen zur Populat ionsdynamik des Crustaceenplanktons im tropischen Lago Castanho (Amazonas, Brasilien). Thèse de Doctorat, Université de Kiel., 109 p.

Brandorff (G.-O.) 1978. Preliminary comparison of the crustacean plank ton of a white and a black water lake in Central Amazon. Verh, int. Ver. Limmol., 20 : 1198-1202.

Brandorff (G.O.), Koste (W.) \& Smirnov (N.-N.) 1982. The composition and structure of Rotifera and crustacean communities of the Lower Rio Nhamundà, Amazonas, Brazil. Stud. Neotrop. Fauna and Envir, $17: 69-121$

Brehm (V.) 1937. Brasilianische Cladoceren. Gesammelt von Dr. O. Schubart. Zweiter Bericht. Int. Rev. d. ges. Hydrobiol. u. Hydrogr., $35: 497.512$.

Brehm (V.) 1953. Eine eigenartige Bosmina aus Venezuela. Ergebnisse der deutschen limnologischen Venezuela Expedition 1952. Anz öst. Akad. Wiss. Wien, 90: 214-217.

Brehm (V.) 1956. Cladocera aus Venezucla. Ergeb. deutsch. limnol. Venezuela-Exp. 1: 217-232.

Brehm (V.) I957. Cladoceren aus Venezuela, zugleich Betrachtungen über vermeintliche und wirkliche Species. Trennung bei Cladoceren. Anz Marh. nat. öst. Akad. Wiss., 11 : 1-13.

Brehrn (V.) \& Thomsen (R.). 1936. - Brasilianische Phyllopoden und Arguliden gesammelt von Herm Dr. O. Schubart. Zool. An: $116: 211-218$.

Brooks (J.L.) 1966. Cladocera in Freshwater Biology, $\left(4^{\circ} \mathrm{ed}\right.$.): 58.7-656. H.B. Ward \& G.C. Whipple, eds.

Carvatho (M.-L.) 1984. Influence of predation by fish and water tur. bidity on a Daphmia gessneri population in an Amazonian floodplain lake, Brasil. Hydrobiologia, 113 : 243-247.

Chien (S-M.) 1970. Alonella fitzpatricki sp. n. and A. leei sp. n. : new Cladocera from Mississippi. Trans. Amer. Micros. Soc., 89 : 53.2.538.

Collado (C.), Femando (C.-H.) \& Sephton (D.) 1984. The freshwater zuoplankton of Central America and the Caribbean. Hydrobio logic, $113: 105-119$.
Daday (E. von). 1905. Untersuchungen über die Süsswasser Mikrofauna Paraguavs. VII. Cladocera. Zoologica, 18 (44) : 1.374.

Delachaux (T, 1917. Cladocères de la région du lac Victoria Nyanza. Rev: Susse de Zool., 25 (3) : 77-93.

Delachaux (T.) 1918. Cladocè res des Andes péruviennes. Bull. Soc. Neuchatel. Sci. Nat., $43: 18-33$.

Durnont (H.J.) 1981. Cladocera and free - living Copepoda from the Fouta Djalon and adjacent mountains areas in West Africa. Hydrobiologia, 85 : $97-116$.

Fernando (C.-H.) 1980. The freshwater zooplankton of Sri Lanka, with a discussion of tropical freshwater zooplankton composition. Int. Revue. ges. Hydrobiol., 65 (1) : 85-125.

Flössner (D.) 1963. Scapholeberis microcephala (Lilljeborg Ms) G.O. Sars 1890 und Alona karelica Stenroos 1897 (Crustacea, Cladocera) in Norddeutschland gefunden. Zool. Anz., $170(5 / 6)$ : 253.254.

Flössner (D.) 1972. Krebstiere, Crustacea. Kiemen und Blattfüsser, Branchiopoda, Fischläuse, Branchiura. Die Tienwell Deutschlands, 60 Teil. $501 \mathrm{p}$.

Frey (D.G.) 1961. Differenciation of Alonella acutrostris (Birge, 1879) and Alonello mostrata (Koch, 184 I) (Cladocera, Chydoridae). Trans. Amer. Microsc. Soc, 80 (2) : 129.140.

Frey (D.G.) 1982. Relocation of Chydorus barroisi and related species (Cladocera, Chydoridae) to a new genus and description of two new species. Hydrobiologia, 86:231-269

Furch (K.) 1984. Water chemistry of the Amazon basin : the distribution of the chemical elements among freshwaters, 167-199. In : The Amazon. Limnology and Lanscape ecalogy of a mighty tropical river and its basin. W. Junk Publishers, Pays-Bas, $\mathrm{H}$. Sioli, ed.

Green (J.) 1962. Zooplankton of the River Sokoto. The Crustacea Proc. Zool. Soc. London, $138: 415-453$.

Harding (J.-P.) 1955. The Percy Sladen Trust Expedition to Lake Titicaca in 1937. XIX. Cristacea : Cladocera. Trans. LiHn Soc. London, 1 (3): 329.354.

Harding (J .P.) \& Petkovski (T.) 1963. Latonopsis australis Sars (Cladocera) in Jugoslavia with notes on its synonymy and distribution. Crustaceana, $6: 1-4$.

Hardy (E.-R.), Robertson (B.) \& Koste (W.) 1984. About the relation ship between the zooplankton and fluctuating water levels of Lago Camaleao, a Central Amazonian vàrzea lake. Amazortiana, 9 (1) : 43-52.

Henry (M.). 1919. On some australian Cladocera. Proc. Roy. Soc. New' Soulh Wales, $52: \mathbf{4 6 3 . 4 8 5}$.

Heredia-Seixas (M.) 1981. Aspectos ecológicos das populaçoes de Cladocera (Crustacea) na represa do Lobo (Broa), S. Carlos, S. Patulo. Thèse de l'Université Fe. S. Carlos, $156 \mathrm{p}$

Herbst (H.-V.) 1967. Copepoda und Cladocera (Crustacea) aus Südamerika. Gewässer und Abwässer, 44/45: 96-108.

Hertst (H.V.) 1968. Diaphanosoma dentatum n. sp. (Crustacea, Cladocera) aus Venezuela. Gewässer tand Abwässer, 46: 7-11.

Herbst (H.V.). 1974. Zwei seltene Chydoridae (Cladocera) am Niederrhein. Gewässer und Abwässer, 53-54:133-137.

Herbst (H.V.) 1975. Diaphanosoma spinulosum n. sp. (Crustacea, Cladocera) aus Venezuela. Gewässer und Abwässer, 57/58: 147.150

Idris (B.A.G.) 1983. Freshwater Zooplankton of Malaysia (Crustacea : Cladocera). Penerbit Universiti Pertanian Malaysia, Serdang, Selangor, Malaysia: 1.153.

Infante (A.) 1980. Los Cladoceros del Lago de Valencia. Acta Cient. Venezolana., $31: 593-603$.

Infante (A.) 1984. A note about Daphnia in Venezuelan waterbodies. Hydrobiologia, 119:81-82.

Kiser (R.W.W) 1948. Two new species of Alona from the Peari River Canton, China. Trans. Amer. Micros. Soc., 68:315-318.

Korovchinsky (N-M.) [985. Sarsilatona, a new genus of the Family Sididae (Crustacea, Cladocera) with two redescribed species and one new species. Int. Rev. ges. Hydrobiol., 70 (3) : 397425 
Korovchinsky (N.-M.) 1983. Genus Lalonopsis, genus Pseudosida. in : N.N. Smirnov and B.V. Timms: A revision of the Austra lian Cladocera (Crustacea), Records of the Australian Museum (1983). Suppl. 1:5-10.

Lewis Jr. (W.-H.) \& Weibezahn (F.) 1981. The chemistry and phyto plankton of the Orinoco and Caroni rivers, Venezuela. Arch. Hydrobiol., 91 : $521-528$.

Lilljeborg (W.) 1900 . Cladocera Sueciae Nova Acta Soc. Sci. Upsala (Sér. 3) : 701 p. Facsimile Reissue of the original Edition with a Prologue, (1982), Rodhe (W.) and Frey (D.G.), eds.

Margalef (R.) 1961. La vida en los charcos de agua dulce de Nueva Esparta (Venezuela). Mem. Soc. Ctenc. Nat. La Salle, 59 (21): $75-110$.

Martinez de Ferrato (A.) 1966. Nuevos Cladòceros para las aguas argentinas. I. Physio. Rev. Soc. Arg. Cienc. Nar. (Buenos Ayres), $26(72): 397-403$

Martinez de Ferrato (A.) 1967. Nuevos Cladóceros para las aguas argentinas. II. Acta. Zool. Lilloana, 23 . 325-330.

Mop. 1979. Atlas de Venezuela. Cartografia Nacional, Caracas. $331 \mathrm{p}$.

Negrea (S.) 1983. Cnustacea, Cladocera. Fauna Republicii Socialiste România, 4 (12): 1.399

Németh (A.), Paolini (J.) \& Herrera (R.) 1980. Carbon transport in the Orinoco river : preliminary results. In : Transport of carbon and mine rals in major rivers. Degens, E.T. (ed.) Mitt. Geol. Palaont. Inst. Univ. Hamburg. SCOPE/UNEP Sonderband, 52 : 357-364.

Olivier (S.R.) 1962. Los Cladoceros argentinos con claves de las especies, notas biologicas y distribution geographica. Rev. Mus. La Plata. (N.S.) Sec. Zool., 7 (56) : 173.269.

Orghidan (T.) \& Negrea (S.). 1970. Quelques Cladocères des eaux souterraines et épigées de Cuba. Trav. Inst. Spéol. «E. Racovitza $\Rightarrow, 9: 113-120$.

Paggi (J -C.) 1972. Nota sistematica acerca de algunos Cladoceros del genero Chydorus Leach 1843, de la Republica Argentina. Physio, 31 (82) : 223-236

Paggi (J.C.) 1979. Aportes al conocimiento de la Fauna argentina de Cladoceros. Il Sobre Alona eximia Kiser, 1948. Neotropica, $25(74)$ : $177-185$

Pearse (A.-S.) 1921. Crustacea from Lake Valencia, Venezuela. Proc. U.S. Nat. Mus., $59: 459-462$.

Pérez (L.) 1984. Ecologia y factibilidad de cultivo de los Engraulidae dulceacuicolas del rio Orinoco. Mems. Asoc. Latinoam. Acuicult., 5 (3) : 533-541.

Ravelo (U.) 1986. Tècnica preliminar de cultivo masivo de cladòceros en condiciones tropicales. Bol. Asoc. Ven. Acuicult. (Sous presse).

Rey (J.) \& Saint-Jean (L.). 1968. Les Cladocères (Crustacés, Branchiopodes) du Tchad. (Premiere note). Cah. O.R.S.T.O.M., sér. Hydrobiol, 11 (3-4) : 79-118.

Rey (J.) \& Vasquez (E.) 1986. Contribution à la connaissance des Cladocères néotropicaux : redescript ion de Leydigiopsis omata Daday, 1905. (Crustacea, Cladocera). Annls Limnol, 22 (2): 169.176.

Rey (J.) \& Vásquez (E.). 1986. Note taxonomique sur Camptocereus dadayi Stingelin, 1913, comb. nov. (Crustacea, Cladocera). Annls Limnol., 22 (2) : 177-180.

Richard (J.) 1897. Entomostracés de l'Amérique du Sud recueillis par M.M.U. Deiters, H. Von Ihering. G.W. Müller et C.O. Poppe. Mem. Soc. Zool. France, $10: 263-301$

Robertson (B.-A.) \& Hardy (E.-R.) 1984. Zooplankıon of Amazonian. lakes and rivers, p. 337-352. In ; The Amazon, Limnology and landscape ecology of a mighty tropical river and its basin. Junk (W.) Publishers, Pays-Bas, Sioli (H.) ed.

Saint.Paul (U.) 1984, Ecological and physiological investigations of Colossoma macropomum, a new species for fish culture in Amazonia. Mems. Asoc. Latinoam Acuicult, 5 (3) : $501-518$.
Sars (G.-O.) 1888. Additional notes on australian Cladocera, raised from dried mud. Forhandl. Vidensk. Selsk. Christiana, $7: 3.74$

Sars (G.-O.) 1901. Contributions to the knowledge of the freshwater Entomostraca of South America, as shown by artificial hatching from dried material. Part I. Cladocera, Arch. Math Nalur: B., 23: 1-102.

Schmidt (G. W.) 1972. Chemical properties of some waters in the tropical rain-forest region of Central-Amazonia along the new road Manaus-Caracari. Amazoniana, 3 (2) : 199.207.

Sendacz (S.) 1984. A stydy of the zooplankton community of Billings Reservoir-Sao Paulo. Hydrobiologia, 113:121-127.

Sioli (H.) 1950. Das Wasser im Amazonasgebiet. Forsch. Fortschr., $26: 274.280$

Sioli (H.) 1951. Zum Alterungsprozess von Flüssen, und Flusstypen im Amazonasgebie1. Arch. Hydrobiol., 43: 267.283.

Sioli (H.) 1956. Uber Natur und Mensch im brasilianischen Amazonasgebiet. Erdkunde, $10: 89-109$.

Sioli (H.) 1965. Bemerkung zur Typologie amazonischer Flüsse. Amazoniana, 1: 74-83.

Smirnov (N.N.) 1971. Chydoridae of the world's fauna. Fauna of the USSR, Crustacea, 1 (2), new series, $n^{\circ} 101$, Leningrad. 531 p. (in Russian). English transl. A. Mercado, Israel Prog. Sci. Transl., 1974.

Smirnov (N.-N.) 1984. Some comments on tropical lit toral Clado cera, with a description of Alona incredibilis sp. nov. Hydro biologia, 113 : 155-158.

Smirnov (N.-N.) \& Tirmm (B.-V.) 1983. A revision of the australian Cladocera (Crustacea). Records of the australian Museum. Suppl. $1: 1.132$.

Stingelin (T.) 1904. Entomostraken gesammelt von Dr. G. Hagmann in Mündungsgebiet des Amazonas. Zool. Jb. Abt. System., 20 : $575-590$.

Stingelin (T.) 1906. Cladoceren aus Paraguay. Zweiter Beitrag zur Kenntnis südamerikarischer Entomostraken. Ann. Biol. Lac., $1: 181.192$

Stingelin (T.) 1913. Cladoceren aus den Gebirgen von Kolumbien. Mem. Soc. netichatel. Sci. Nat, $5: 600-638$.

Thomas (1..F.) 1961. Review of the genera Pseudosida Herrick, 1884 and Latonopsis Sars, 1888 (Cladocera). Cristaceana, 3 (1): 1.8.

Thomasson (K.) 1953. Studien über das südannerikanische Süsswas serplankton. 2. Zur Kenntnis südamerikanischen Zooplankton. Arkiv för Zool, sér. 2, 6: 189-194.

Van de Velde (I.), Dumont (H.-J.) \& Grootaert (P.) 1978. Report on a collection of Cladocera from Mexico and Guatemala. Arch. Hydrobiol, $83: 391.404$.

Vàsquez (E.) 1984. El zoopiancton de la secciòn baja de un rìo de aguas negras (rio Caroni) y de un embalse hidroeléctrico (Macagua), Venezuela. Mem. Soc. Cienc. Nat. La Salle, 44 (121) 109-129.

Vàsquez. (E.) \& Sanchez (L.) 1984. Variacion estacional del planc$t o n$ en dos sectores del rio Orinoco y une laguna de inundacion adyacente. Mem. Soc. Cienc. Nat. La Salle, 44 (121) : 11-31.

Zago (M.S.A.) 1976. The planktonic Cladocera (Crustacea) and aspects of the eutrophication of Americana Reservoir, Brazil Bolm. Zool. Univ. S. Paulo, 1 : 105-145.

Zinck (A.) 1982. Rios de Venezuela. Cuadernos Lagovèn. Caracas. $63 \mathrm{p}$.

Zoppi de Roa (E.). 1972. Zooplankton de la laguna de Campona, Edo. Sucre, Venezuela. Cuad. Oceanogr. Univ. Oriente, $3: 49-53$.

Zoppi de Roa (E.). Los cladoceros de las sabanas inundables de Mantecal (Venezuela). Bol. Soc. Ven. Cienc. Nat., (sous presse). 